\title{
A TARGETED SEARCH FOR PECULIARLY RED L AND T DWARFS IN SDSS, 2MASS, AND WISE: DISCOVERY OF A POSSIBLE L7 MEMBER OF THE TW HYDRAE ASSOCIATION
}

\author{
Kendra Kellogg ${ }^{1,2,6}$, Stanimir Metchev ${ }^{1,2}$, Kerstin Geißler ${ }^{2}$, Shannon Hicks ${ }^{2}$, J. Davy KirkPatrick ${ }^{3}$, and \\ RADOSTIN KURTEV ${ }^{4,5}$ \\ ${ }^{1}$ Western University, Centre for Planetary and Space Exploration, 1151 Richmond St, London, ON N6A 3K7, Canada; kkellogg@uwo.ca, smetchev@uwo.ca \\ 2 Stony Brook University, Stony Brook, NY 11790, USA \\ ${ }^{3}$ Infrared Processing and Analysis Center, Mail Code 100-22, California Institute of Technology, 1200 E. California Blvd., Pasadena, CA 91125, USA \\ ${ }^{4}$ Instituto de Física y Astronomía, Facultad de Ciencias, Universidad de Valparaíso, Ave. Gran Bretaña 1111, Playa Ancha, Casilla 53, Valparaíso, Chile \\ ${ }^{5}$ Millennium Institute of Astrophysics, Chile \\ Received 2014 October 10; accepted 2015 October 22; published 2015 November 24
}

\begin{abstract}
We present the first results from a targeted search for brown dwarfs with unusual red colors indicative of peculiar atmospheric characteristics. These include objects with low surface gravities or with unusual dust content or cloud properties. From a positional cross-match of SDSS, 2MASS, and WISE, we have identified 40 candidate peculiar early-L to early-T dwarfs that are either new objects or have not been identified as peculiar through prior spectroscopy. Using low-resolution spectra, we confirm that 10 of the candidates are either peculiar or potential $\mathrm{L} / \mathrm{T}$ binaries. With a $J-K_{s}$ color of $2.62 \pm 0.15 \mathrm{mag}$, one of the new objects-the L7 dwarf 2MASS J11193254-1137466-is among the reddest field dwarfs currently known. Its proper motion and photometric parallax indicate that it is a possible member of the TW Hydrae moving group. If confirmed, it would be the lowest-mass (5-6 $M_{\text {Jup }}$ ) free-floating member. We also report a new T dwarf, 2MASS J22153705+2110554, that was previously overlooked in the SDSS footprint. These new discoveries demonstrate that despite the considerable scrutiny already devoted to the SDSS and 2MASS surveys, our exploration of these data sets is not yet complete.
\end{abstract}

Key words: binaries: close - brown dwarfs - infrared: stars - stars: late-type - stars: peculiar

\section{INTRODUCTION}

Compared to main sequence stars, ultra-cool dwarfs display a wide range of near-infrared (near-IR) colors, even among objects at the same effective temperature or spectral type. The diversity is diagnostic of the unique processes taking place in their molecule- and condensate-rich atmospheres. Effective temperature is the main factor that governs the photospheric appearance of field-aged brown dwarfs, with current understanding pointing to a monotonic correspondence between effective temperature and optical spectral type (Golimowski et al. 2004; Vrba et al. 2004; Looper et al. 2008a).

Cruz et al. (2009) proposed a dimensional extension to the classification scheme for brown dwarfs by incorporating surface gravity as a second parameter. They adopt a qualitative description of surface gravities-intermediate, low, and very low-based on optical spectral line strengths. Allers \& Liu (2013) expanded the classification scheme to the near-IR by adding continuum index measures to classify the absorption strengths of volatile molecules.

Low surface gravities generally contribute to higher dust content in the upper atmospheres of brown dwarfs, making them redder. Analyses of the $\mathrm{L}$ and $\mathrm{T}$ dwarf population have shown that the optical and near-IR colors of low surface gravity objects are readily distinguishable from those of "normal" objects (e.g., Knapp et al. 2004; Cruz et al. 2009; Faherty et al. 2012; Allers \& Liu 2013). However, there is also evidence of red brown dwarfs with high dust content without any signatures of youth (Looper et al. 2008b; Kirkpatrick et al. 2010). Their near-IR colors are very similar to those of the young, low surface gravity objects, but their spectra do not have any of the

\footnotetext{
${ }^{6}$ Visiting Astronomer at the Infrared Telescope Facility, which is operated by the University of Hawaii under contract NNH14CK55B with the National Aeronautics and Space Administration.
}

characteristics of youth. That is, peculiarly red brown dwarfs may not necessarily be low gravity and hence young, but could instead be unusually dusty. As there have not been many unusually red old L dwarfs found, the cause of such dustiness is not well established.

Finding the cause of the enhanced dust content is undoubtedly of interest for understanding the evolution of substellar objects and the processes that affect the sedimentation and/or condensation of atmospheric dust. It is also crucial for revealing the ages and properties of directly imaged extrasolar planets, most of which exhibit spectral energy distribution (SED) characteristics of both youth and high dust content (e.g., Marley et al. 2012; Bonnefoy et al. 2013). Because isolated brown dwarfs can be scrutinized much more readily than directly imaged extrasolar planets, we stand to potentially learn more about ultra-cool atmospheres from brown dwarfs than we can from exoplanets.

Our understanding of the nature of brown dwarfs with unusual SEDs is presently hindered by the relatively small numbers of such peculiar objects. Until recently, there have been no color-selected searches for peculiar brown dwarfs. Discoveries have been serendipitous, usually a by-product of searches for T dwarfs (Burgasser et al. 2004; McLean et al. 2007; Looper et al. 2008a, 2008b, etc.). Only over the past few years have targeted searches been performed on large-area surveys to specifically seek unusually red objects (e.g., Aller et al. 2013; Gagné et al. 2015).

In view of this, we are conducting an independent program to find $\mathrm{L}$ and $\mathrm{T}$ dwarfs with unusual optical/near-IR colors. The goal is to substantially expand the sample of peculiar $\mathrm{L}$ and $\mathrm{T}$ dwarfs in order to map the full range of their photospheric properties and to better understand the evolution and content of their atmospheres. We cross-correlated the SDSS, 2MASS, and WISE survey databases to seek candidate peculiar brown 
dwarfs based solely on photometric criteria. Our first pass through the databases focused mainly on identifying unusually red objects. Most notable among these is one of the reddest L dwarfs ever found (2MASS J11193254-1137466; 2MASS $J-K_{s}=2.62 \pm 0.15 \mathrm{mag}$ ). While peculiar $\mathrm{L}$ and $\mathrm{T}$ dwarfs have until now been found mostly serendipitously in largescale photometric surveys, we have implemented a systematic approach to find these objects by design. We discuss the selection and prioritization of candidates in Section 2 and their follow-up observations in Section 3. The spectroscopic characterization of the new $\mathrm{L}$ and $\mathrm{T}$ dwarfs is presented in Section 4. In Section 5 we assess the significance of the findings from our systematic search of peculiar objects in the context of the presently known sample of $\mathrm{L}$ and $\mathrm{T}$ dwarfs.

\section{CANDIDATE SELECTION}

We employ a photometric search for peculiar L and T dwarfs using combined optical (SDSS), near-IR (2MASS), and mid-IR (WISE) fluxes. Our candidate selection expands on the procedure presented in Metchev et al. (2008) and Geißler et al. (2011), which applied joint positional and color constraints to search for $\mathrm{T}$ dwarfs in the overlap area of 2MASS and SDSS DR1 $\left(2099 \mathrm{deg}^{2}\right)$. We use the ninth Data Release (DR9) from SDSS (Ahn et al. 2012), which has a $14,555 \mathrm{deg}^{2}$ footprint, encompassing an area 6.9 times larger than the DR1 footprint. The $>10$ year observational epoch difference between 2MASS and SDSS DR9 prompts us to choose a much larger cross-match radius than was used in the first two studies. We used the Virtual Astronomical Observatory catalog cross-comparison tool and chose a cross-match radius of 16 ". 5 to maintain sensitivity to objects with proper motions as high as $1 . " 5 \mathrm{yr}^{-1}$.

\subsection{Selection Criteria}

Our magnitude and color selection criteria are summarized below. In the following, all griz magnitudes are on the SDSS photometric scale (Lupton et al. 2002), and the 2MASS and WISE magnitudes are on the Vega scale:

1. $z-J>2.5 \mathrm{mag}$

2. $i-z>1.5 \mathrm{mag}$;

3. $J>14 \mathrm{mag}$;

4. $z \leqslant 21 \mathrm{mag}$ and $z_{\mathrm{err}} \leqslant 0.2 \mathrm{mag}$;

5. no $g, r<23$ mag detection within 1 !' 3 of the 2 MASS coordinate;

6. SDSS object flag setting type $=6$ or 3 (point or extended source);

7. 2 MASS object flag setting mp_flg $=0$ (i.e., not marked as a known minor planet), gal_contam $=0$ (i.e., not contaminated by a nearby 2MASS extended source), and ext_key $=$ NULL (i.e., not extended in 2MASS);

8. $H-W 2>1.2 \mathrm{mag}$

9. $z-J>-0.75\left(J-K_{s}\right)+3.8 \mathrm{mag}$ (criterion used only to prioritize follow-up of red outliers).

Our $z-J$ and $i-z$ color cuts (criteria 1 and 2) were chosen to ensure sensitivity to $\mathrm{L}$ and $\mathrm{T}$ dwarfs, all of which have a steep red optical slope. The $J>14$ mag cutoff was imposed to minimize the large number of candidates representing the cross-identification of a bright star artifact in SDSS (e.g., a filter glint or a diffraction spike, especially near saturated stars) with the (unsaturated) image of the same star in 2MASS. Criterion 4 was chosen to ensure detection in SDSS with at least a moderate $\mathrm{S} / \mathrm{N}$.

Our 16". 5 matching radius commonly resulted in multiple matches of nearby faint SDSS objects to the same, brighter 2MASS object. Each of these individual matches would nominally satisfy the color and magnitude selection criteria, since the faint SDSS photometry would be paired with the brighter 2MASS photometry. However, visual inspection clearly demonstrated that the SDSS and 2MASS objects were distinct, and that the actual object in SDSS that positionally matched the 2MASS object was not nearly as red, and so did not satisfy the $z-J>2.5 \mathrm{mag}$ criterion. Therefore, we discarded any object that had a $g$-band detection (i.e., $g \leqslant 23$ mag and likely a star) in the SDSS catalog within 1". 3 (the angular resolution of SDSS) of the original 2MASS coordinates (criterion 5 ). This removed $\sim 86 \%$ of the candidate sample.

The SDSS object flag restrictions (criterion 6) ensure that the identified candidates are not known artifacts or flux measurements of the blank sky in SDSS. The SDSS morphological star-galaxy separation is $<97 \%$ accurate for $r \geqslant 21 \mathrm{mag}$ (Yasuda et al. 2001) so we include both star and galaxy object types in this criterion in case our faint brown dwarfs were misclassified. We also wanted to ensure that they are not known minor planets, extended or contaminated by nearby extended sources in 2MASS (criterion 7).

To make sure that all of the objects in our candidate list were real objects, we cross-matched our list with the WISE All-sky Data Release using the SDSS coordinates. Our objects are expected to be detected in the WISE W1 band because outside of the galactic plane the $W 1 \mathrm{~S} / \mathrm{N}=5$ level corresponds to $\lesssim 16.8$ mag. ${ }^{7}$ This matches the 2 MASS $K_{s}$ flux limit at high galactic latitude, especially since $\mathrm{L}$ and $\mathrm{T}$ dwarfs have positive $K_{s}-W 1$ colors. A radius of 16 " 5 was again chosen for this cross-match. An additional color cut was applied on $H-W 2$ (criterion 8) in order to select only $\mathrm{L}$ and $\mathrm{T}$ dwarfs (based on the color-spectral type relations from Kirkpatrick et al. 2011). This removed $\sim 74 \%$ of the remaining sample.

Finally, we visually inspected the images of remaining candidates using the Infrared Science Archive Finder Chart service $^{8}$ and removed objects that were contaminated by nearby extended sources in SDSS. This eliminated approximately $22 \%$ of the remaining candidate sample, leaving us with 314 candidates (Figure 1(a)).

\subsection{Prioritization of Peculiar Objects}

Since our goal was to select unusually red brown dwarfs in the absence of spectral type information, an additional color criterion (9) was set in order to prioritize red objects. To decide the form of the color criterion, we first analyzed the spectra of $\mathrm{L}$ and $\mathrm{T}$ dwarfs in the SpeX Prism Archive ${ }^{9}$ by forming synthetic photometry over various red optical and near-IR bandpasses. These $\mathrm{L}$ and $\mathrm{T}$ dwarfs with archival SpeX data formed our control sample, based on which we designed our $z-J$ versus $J-K_{s}$ criterion 9 (Figure 1(b)).

Given the available spectral type information, the unusually red objects in the control sample were set to be those for which the $J-K_{s}$ color was $>2 \sigma$ redder than the median for the

\footnotetext{
7 http://wise2.ipac.caltech.edu/docs/release/allsky/expsup/sec6_3a.html

8 http://irsa.ipac.caltech.edu/applications/FinderChart/

9 http://pono.ucsd.edu/ adam/browndwarfs/spexprism/
} 


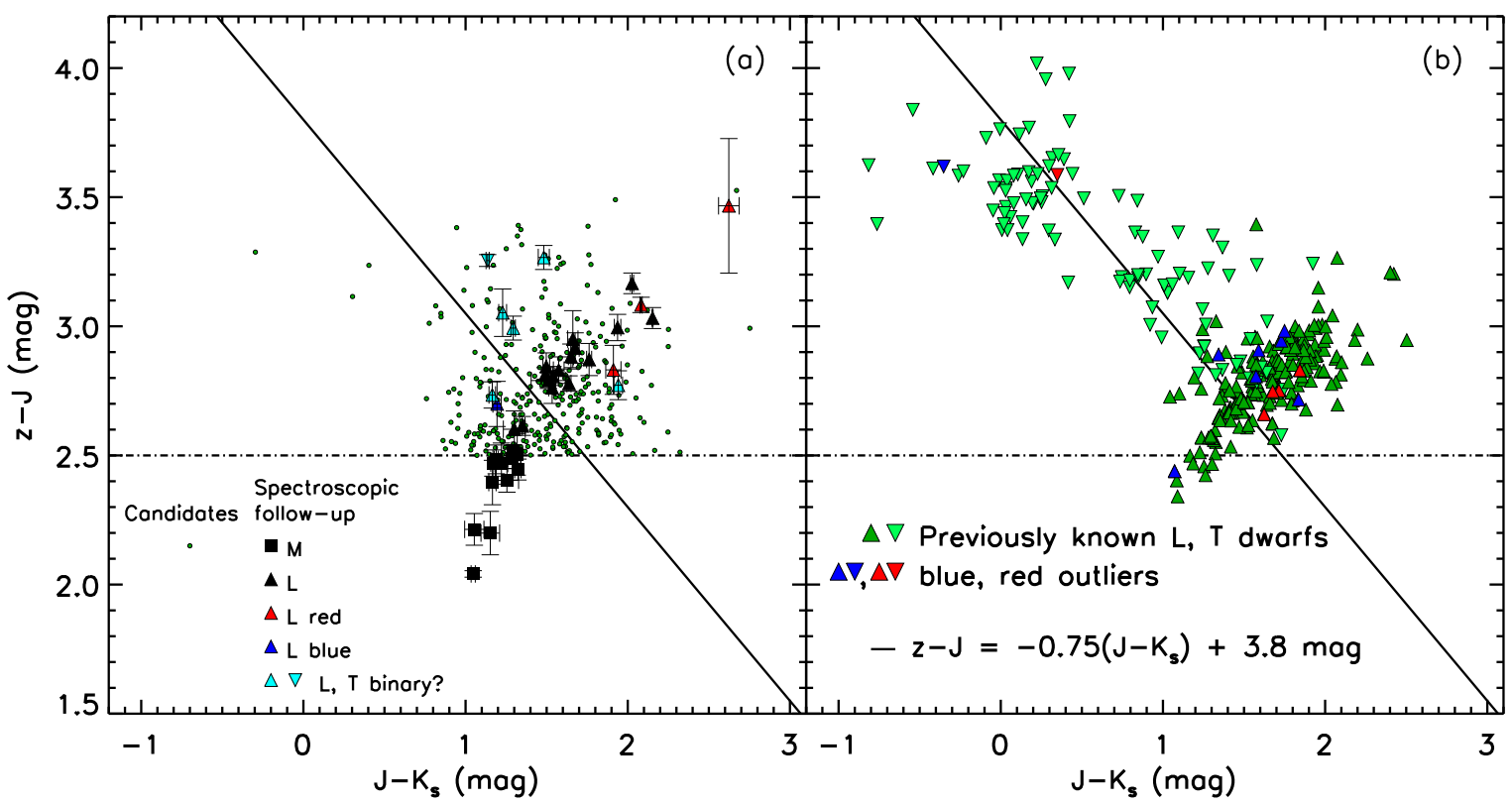

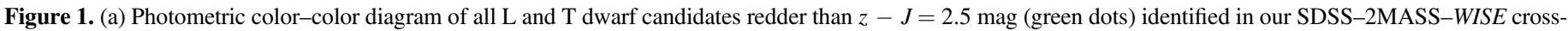

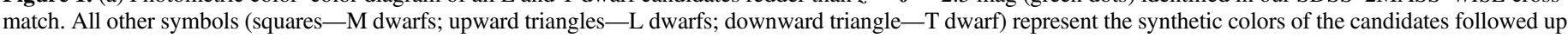

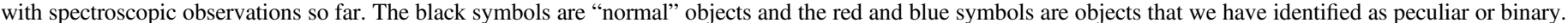

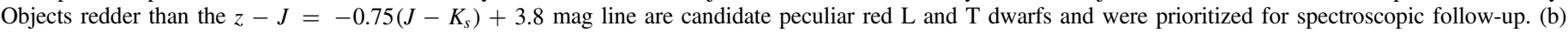

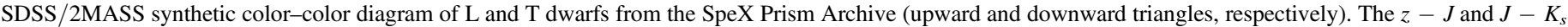

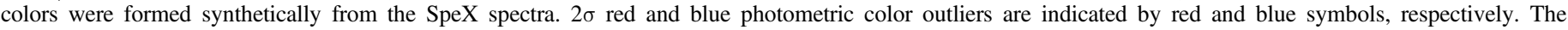
$z-J=-0.75\left(J-K_{s}\right)+3.8$ mag line was designed to select the photometric red outliers.

spectral subtype. The medians and standard deviations of the $J-K_{s}$ colors of M8-T8 dwarfs were adopted from Faherty et al. (2009; M8-M9 and T0-T9) and from Faherty et al. (2013; L0-L9). The unusually red objects in the control sample are shown with red symbols in Figure 1(b). The number of objects from our sample that passed these criteria was 178 .

The color prioritization did not streamline our observational follow-up strategy significantly, as the scatter in colors among spectral types is larger than the scatter at any given spectral type. Nonetheless, we did observe the reddest candidates whenever possible, and included observations of lower-priority targets only as necessary.

\section{SPECTROSCOPIC OBSERVATIONS AND DATA REDUCTION}

Once our candidates were selected, we performed follow-up spectroscopic observations of 40 of the objects $(\sim 13 \%$ of the total candidate sample; 22 high priority and 18 lower priority) using the SpeX instrument (Rayner et al. 2003) on the NASA Infrared Telescope Facility (IRTF) and the Folded-port InfraRed Echellette (FIRE) instrument (Simcoe et al. 2008) on the Magellan-Baade telescope. Conditions were photometric on most nights, except on 2011 August 3 and 2012 April 18, 19 , and July 14, when there was scattered cirrus. All reduction of the low-resolution spectra (SpeX and FIRE low-dispersion (LD)) was done in Interactive Data Language (IDL).

\subsection{IRTF/SpeX}

The majority of our follow-up observations were taken using the SpeX spectrograph on the IRTF. The broad, simultaneous wavelength coverage $(0.8-2.5 \mu \mathrm{m})$ of SpeX and its location in the northern hemisphere are ideal for follow-up of SDSS- identified candidates. These spectra were obtained between 2011 August and 2013 June. The observations were taken in prism mode either with the 0 ". $8 \times 15$ ". 0 or with the 1 !. $6 \times 15$ !" 0 slit, resulting in resolutions of $R \sim 150$ and $\sim 75$, respectively. The slit orientation was maintained to within $20^{\circ}$ of the parallactic angle for all targets. We used a standard A-B-B-A nodding sequence along the slit to record object and sky spectra. Individual exposure times were either 60 or $180 \mathrm{~s}$ per pointing. The shorter exposure times allowed us to better subtract the sky glow under changing atmospheric conditions. Standard stars were used for flux calibration and telluric correction. Flat-field and argon lamps were observed immediately after each set of target and standard star observations for use in instrumental calibrations. Observation epochs and instrument settings for each science target are given in Table 1.

All reductions of the data taken with SpeX were carried out with the SPEXTOOL package version 3.4 (Vacca et al. 2003; Cushing et al. 2004), using a weighted profile extraction approach (Horne 1986; Robertson 1986). The aperture widths were set to be the radius at which the spatial profile dropped to $\sim 5 \%$ of the peak flux value to ensure no contamination from background noise; the background regions were chosen to begin at the edge of the point-spread function (PSF) radius (i.e., beyond 2.5 pixels $=0$ ". 375 ). A constant value was fit to the background and subtracted from the spectrum. The individual extracted and wavelength-calibrated spectra from a given sequence of observations, each with their own A0 standard, were then scaled to a common median flux and were mediancombined using X_COMBSPEC. The combined spectra were corrected for telluric absorption and were flux-calibrated using the respective telluric standards with $x_{-}$TELLCOR. All calibrated sets of observations of a given object were median-combined to produce the final spectrum. The reduced spectra were smoothed 
Table 1

SpeX Observations

\begin{tabular}{|c|c|c|c|c|c|}
\hline $\begin{array}{l}\text { Identifier } \\
\text { (J2000) }\end{array}$ & $\begin{array}{l}\text { Date } \\
\text { (UT) }\end{array}$ & $\begin{array}{c}\text { 2MASS J } \\
(\mathrm{mag})\end{array}$ & $\begin{array}{l}\text { Slit Width } \\
(\operatorname{arcsec})\end{array}$ & $\begin{array}{c}\text { Exposure } \\
\text { (minute) }\end{array}$ & A0 Calibrator \\
\hline 2MASS J08095903+4434216 & 2011 Dec 31 & 16.44 & 0.8 & 24 & HD 75135 \\
\hline 2MASS J09572983+4624177 & 2013 Jun 06 & 16.25 & 1.6 & 24 & HIP 53735 \\
\hline 2MASS J11193254-1137466 & 2013 Jun 06 & 17.29 & 1.6 & 16 & HIP 53735 \\
\hline$\ldots{ }^{\mathrm{a}}$ & 2013 Jun 07 & 17.29 & 1.6 & 8 & HIP 54815 \\
\hline 2MASS J11260310+4819256 & 2013 Jun 07 & 17.20 & 1.6 & 24 & HIP 54815, HIP 56147 \\
\hline 2MASS J14025564+0800553 & 2013 Jun 07 & 16.84 & 1.6 & 160 & HIP 68868, HIP 116886 \\
\hline 2MASS J16005759+3021571 & 2011 Aug 02 & 16.97 & 0.8 & 54 & HD 153650 \\
\hline 2MASS J16094569+1426422 & 2011 Aug 03 & 16.84 & 0.8 & 60 & HD 152531 \\
\hline 2MASS J16091143+2116584 & 2011 Aug 02 & 16.96 & 0.8 & 60 & HD 153650 \\
\hline 2MASS J16135698+4019158 & 2012 Apr 19 & 17.05 & 0.8 & 48 & HD 151353 \\
\hline 2MASS J16231308+3950419 & 2012 Apr 18 & 16.97 & 0.8 & 60 & HD 165623 \\
\hline 2MASS J16370238+2520386 & 2011 Aug 03 & 16.50 & 0.8 & 36 & HD 157359 \\
\hline 2MASS J16403870+5215505 & 2012 Apr 19 & 17.22 & 0.8 & 60 & HD 155838 \\
\hline 2MASS J16410015+1335591 & 2011 Aug 03 & 16.90 & 0.8 & 48 & HD 157359 \\
\hline 2MASS J16470847+5120088 & 2012 Apr 19 & 17.03 & 0.8 & 48 & HD 155838 \\
\hline 2MASS J16592987+2055298 & 2012 Jul 15 & 16.33 & 1.6 & 90 & HD 164728 \\
\hline 2MASS J17081563+2557474 & 2011 Aug 02 & 16.42 & 0.8 & 48 & HD 164728 \\
\hline 2MASS J17145224+2439024 & 2012 Jul 14 & 16.84 & 0.8 & 12 & HD 165623 \\
\hline 2MASS J17161258+4125143 & 2011 Aug 03 & 16.75 & 0.8 & 36 & HD 165623, HD 165622 \\
\hline$\ldots^{\mathrm{a}}$ & 2012 Jul 15 & 16.75 & 1.6 & 36 & HD 165623 \\
\hline 2MASS J17251557+6405005 & 2012 Jul 15 & 16.81 & 1.6 & 24 & HD 165622 \\
\hline 2MASS J17373467+5953434 & 2012 Apr 19 & 16.88 & 0.8 & 60 & HD 166639 \\
\hline 2MASS J21050130-0533505 & 2011 Aug 03 & 16.42 & 0.8 & 36 & HD 209051 \\
\hline$\ldots^{\mathrm{a}}$ & 2012 Jul 14 & 16.82 & 1.6 & 36 & HD 220184 \\
\hline$\ldots^{\mathrm{a}}$ & 2012 Jul 15 & 16.82 & 0.8 & 24 & HD 210265 \\
\hline 2MASS J23023319-0935188 & 2012 Jul 15 & 16.80 & 0.8 & 60 & HD 222903 \\
\hline 2MASS J23322678+1234530 & 2013 Jun 06 & 16.89 & 1.6 & 42 & HIP 68868 \\
\hline 2MASS J23443744-0855075 & 2011 Aug 02 & 16.77 & 0.8 & 60 & HD 2717 \\
\hline
\end{tabular}

Note.

${ }^{\text {a }}$ Repeat observations of the same object, combined with the previous data.

to the instrumental resolution corresponding to the chosen slit width using the Savitzky-Golay smoothing kernel (Press et al. 1992).

\subsection{Magellan/FIRE}

Two of the 40 total candidates were observed using the FIRE spectrograph on the $6.5 \mathrm{~m}$ Magellan telescope. The observations of these objects were taken in LD mode with the 0 " $6 \times 50$ ". 0 longslit, resulting in a resolution of $\sim 400$. We used a standard A-B-B-A nodding sequence along the slit to record object and sky spectra. Individual exposure times ranged from 31.7 to $126.8 \mathrm{~s}$ per pointing, depending on the brightness of the object. Standard stars were used for flux calibration and telluric correction. We used optimal gain settings of $1.2 \mathrm{e}^{-} / \mathrm{DN}$ and $3.8 \mathrm{e}^{-} / \mathrm{DN}$ for the science targets and $3.8 \mathrm{e}^{-} / \mathrm{DN}$ for the standards as suggested in the FIRE observing manual. ${ }^{10}$ Illumination and appropriate pixel flats were observed either at the beginning or the end of the night and a neon-argon lamp was observed immediately after each set of target and standard star observations for use in instrumental calibrations. All science and telluric observations were taken using the sampleup-the-ramp readout mode, whereas all calibration observations were taken in Fowler 1 mode due to the shortness of the exposure times. Observation epochs and instrument settings for each target are given in Table 2.

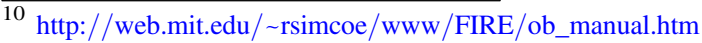


Table 2

FIRE Observations

\begin{tabular}{lcccccc}
\hline \hline $\begin{array}{l}\text { 2MASS ID } \\
\text { (J2000) }\end{array}$ & $\begin{array}{c}\text { Date } \\
(\mathrm{UT})\end{array}$ & $\begin{array}{c}J \\
(\mathrm{mag})\end{array}$ & $\begin{array}{c}\text { Dispersion } \\
\text { Mode }\end{array}$ & $\begin{array}{c}\text { Slit Width } \\
(\mathrm{arcsec})\end{array}$ & $\begin{array}{c}\text { Gain } \\
\left(\mathrm{e}^{-} / \mathrm{DN}\right)\end{array}$ & $\begin{array}{c}\text { Exposure } \\
(\mathrm{minute})\end{array}$ \\
\hline 2MASS J07483864+1743329 & 2012 Mar 21 & 16.27 & Long Slit & 0.60 & 3.8 & $\begin{array}{c}\text { A0 Calibrator } \\
\text { 2MASS J16110632+0025469 }\end{array}$ \\
\hline
\end{tabular}

The FIRE LD spectra were reduced using the FIREHOSE Low Dispersion package, which evolved from the optical echelle reduction software package MASE (Bochanski et al. 2009). The spectra were extracted using the optimal extraction approach with the aperture radius being the PSF radius (usually $\sim 3$ pixels $=0$ ". 45 ), which was then masked to prevent biasing to the sky model. A local background was modeled using a basis spline (i.e., piecewise polynomial) fit to the masked profile and subtracted from the spectra, which were subsequently extracted using a weighted profile extraction approach (Horne 1986). The extracted spectra were wavelength-calibrated and each set of observations was mediancombined. The combined spectra were corrected for telluric absorption and flux-calibrated with their associated A0 calibration star. All calibrated sets of observing sequences of a given object were median-combined to produce a final spectrum. The reduced spectra were smoothed, using the IDL Savitzky-Golay smoothing algorithm, to the same resolution as the SpeX standards for comparison.

\subsection{Synthetic Photometry}

While comparing the 2MASS colors of our L and T dwarf candidates to their spectra, we noted that in a significant fraction of cases the 2MASS colors were too red compared to the spectra. All of our objects were flux-calibrated with A0 stars with known $B-V$ colors, observed at similar airmasses, so we had no reason to suspect a chromatic effect in our flux calibration. Instead, the reason for the discrepancy was traced to a flux overestimation bias at low $\mathrm{S} / \mathrm{N}$ in 2MASS.

Our objects are faint and often near the $\mathrm{S} / \mathrm{N}=5$ detection limit of 2MASS in the $J$-band filter. The greater noise near the detection limit means that objects that would normally be below the limit have a finite chance of appearing brighter because of statistical variations. The effect enhances the number of faint objects with low $\mathrm{S} / \mathrm{N}$ in a flux-limited survey, becoming increasingly important at $\mathrm{S} / \mathrm{N}<10$ (Cutri 2006). Because all of our objects are faint and red, their 2MASS $J$ band magnitudes preferentially suffer from this bias, resulting in redder than expected $z-J$ colors. This effect is particularly large in the case of the few faint $\mathrm{M}$ dwarfs that entered our sample because of their biased photometric colors (Section 4.1). Figure 2 shows how the synthetic colors compare to the photometric colors as a function of the photometric $J$-band $\mathrm{S} / \mathrm{N}$ for both $z-J$ and $J-K_{s}$. Indeed, at lower $\mathrm{S} / \mathrm{N}$, the $z-J$ photometric colors are on average redder than their synthetic colors while the $J-K_{s}$ photometric colors are on average slightly bluer.

For the remainder of our analysis we use only synthetic SDSS $z$ and 2MASS $J H K_{s}$ magnitudes for our candidates and for previously known objects with SpeX Prism Archive spectra. The errors on the synthetic photometry in Table 3 are standard errors derived from the scatter among the continuum slopes of the individual 60 or $180 \mathrm{~s}$ exposure

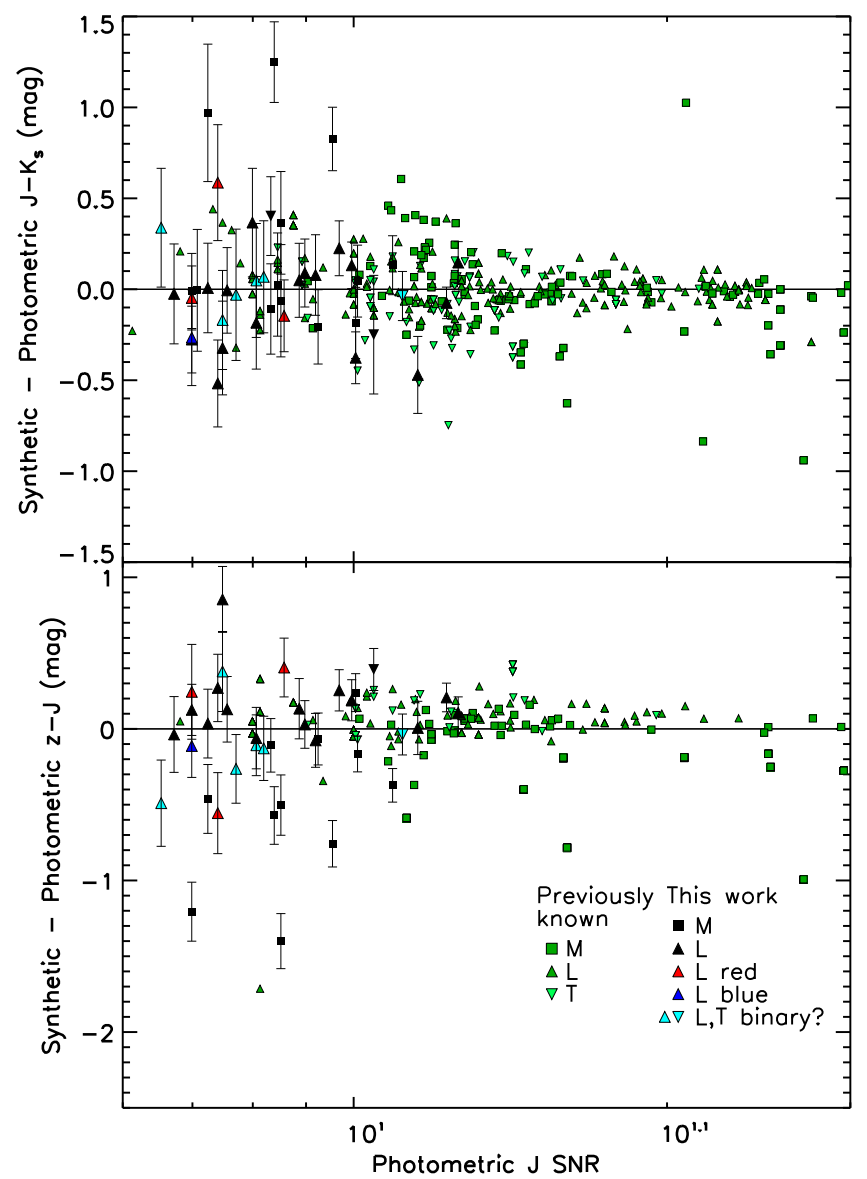

Figure 2. Difference in synthetic vs. photometric $J-K_{s}$ and $z-J$ colors for $\mathrm{M}, \mathrm{L}$, and $\mathrm{T}$ dwarfs from the SpeX Prism Archive (green symbols) and for objects from this work (all other colored symbols). The black symbols are "normal" objects and the blue and red symbols are objects that we have identified as peculiar or binary. Fewer objects appear in the $z-J$ comparison (lower panel) because not all SpeX Archive objects are in the SDSS database.

spectra of our targets and their corresponding standard stars. These errors incorporate systematic uncertainties from potential chromatic slit losses should the targets have been imperfectly positioned on the slit.

\section{SPECTRAL CLASSIFICATION RESULTS}

We estimate spectral types for our objects by comparing them to spectra of brown dwarfs available from the SpeX Prism Archive. ${ }^{11}$ When our spectra do not match any of the normal brown dwarf spectra, we compare to other unusual spectra. In this way, we are able to assess potential spectroscopic peculiarities that may not be evident from the colors alone.

\footnotetext{
11 Kirkpatrick et al. (2010), Burgasser et al. (2004, 2006b, 2007, 2008, 2010), Looper et al. (2007), Chiu et al. (2006), and Reid et al. (2006).
} 
Table 3

Results from Spectroscopic Classification and Synthetic Photometry

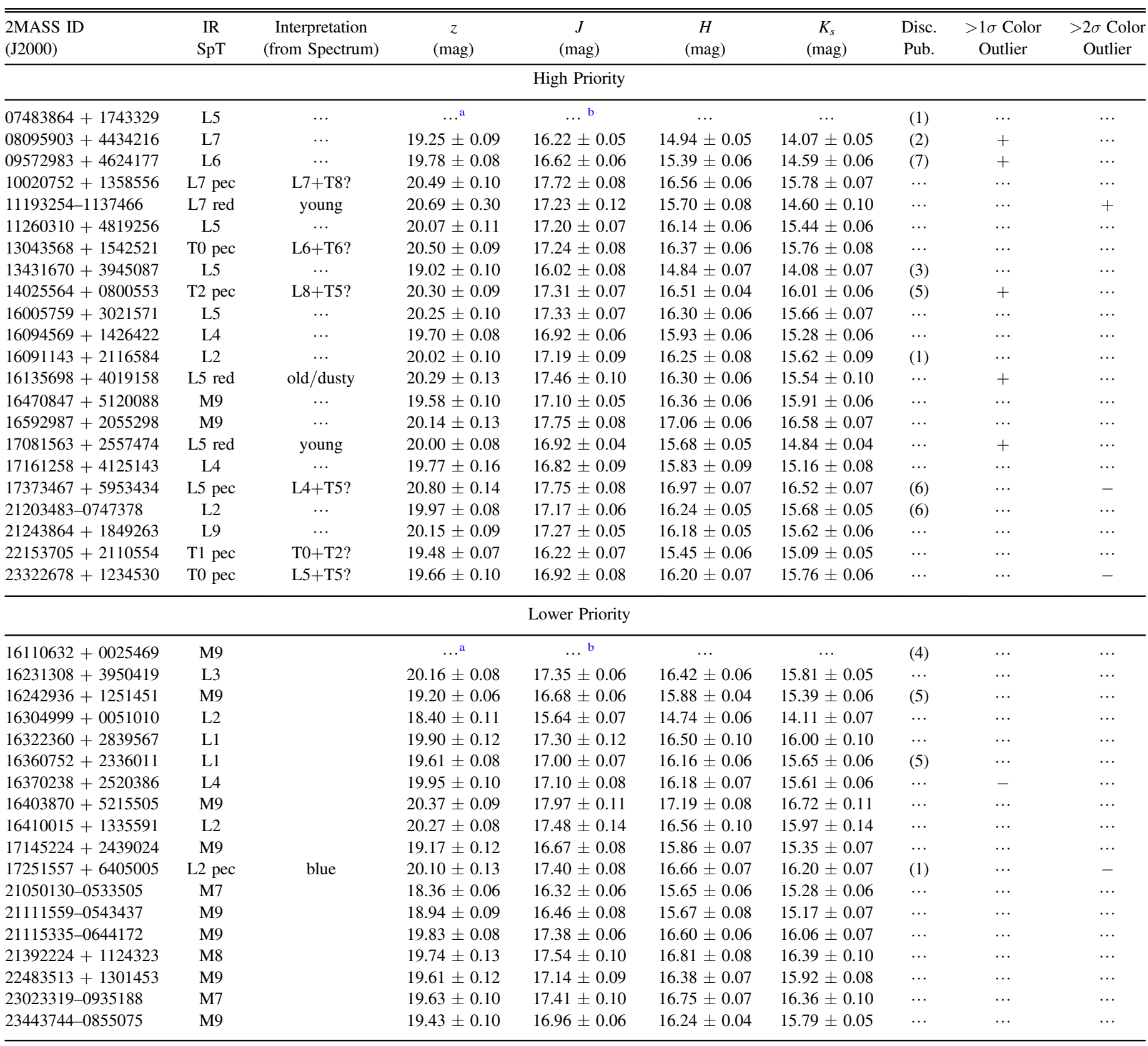

Notes. Determinations of color outliers came from comparing synthetic $J-K_{s}$ colors to average $J-K_{s}$ colors for M8-M9 and T0-T8 spectral types from Faherty et al. (2009) and for L0-L9 spectral types from Faherty et al. (2013). Positives and negatives indicate whether the object was above or below the average, respectively. (1) Zhang et al. (2009), (2) Knapp et al. (2004), (3) Kirkpatrick et al. (2000), (4) Schmidt et al. (2010), (5) Chiu et al. (2006), (6) Geißler et al. (2011), (7) Luhman \& Sheppard (2014).

${ }^{a}$ FIRE spectra do not cover the entire SDSS $z$-band.

$\mathrm{b}$ Target and standard observations were taken with different gain settings so individual $J H K_{s}$ magnitudes are not reported.

Finally, following the approach of Burgasser (2007) and Burgasser et al. (2010), we form combination templates from the standards to assess whether any of our objects might be best fit as unresolved binaries. For spectral comparison to standard $\mathrm{L}$ and $\mathrm{T}$ dwarfs we used $\chi^{2}$ minimization over the $0.95-1.35 \mu \mathrm{m}$ wavelength range. To assess candidate binarity we compare our spectra to combinations of $\mathrm{L}$ and/or $\mathrm{T}$ dwarf doubles over the entire $0.8-2.5 \mu \mathrm{m}$ range, as detailed in Section 4.3. Table 3 lists the determined spectral types, the characteristics of each object, and the peculiarities of our objects determined from both colors and a detailed analysis of their spectra. All of our spectra are shown in Figure 3.

We determined that our candidate list of 40 observed objects includes $13 \mathrm{M}$ dwarfs, $26 \mathrm{~L}$ dwarfs, and $1 \mathrm{~T}$ dwarf. Of these, 10 were previously known and suspected to be $\mathrm{L}$ dwarfs but did not have any published near-IR spectra. The remaining 30 are new, including the $\mathrm{T}$ dwarf. Ten of the $27 \mathrm{~L}$ and $\mathrm{T}$ dwarfs are either peculiar (4) or possible unresolved binaries (6).

The newly classified $\mathrm{M}, \mathrm{L}$, and T dwarfs are plotted on the $z-J$ versus $J-K_{s}$ color-color diagram in Figure 1(a), where 

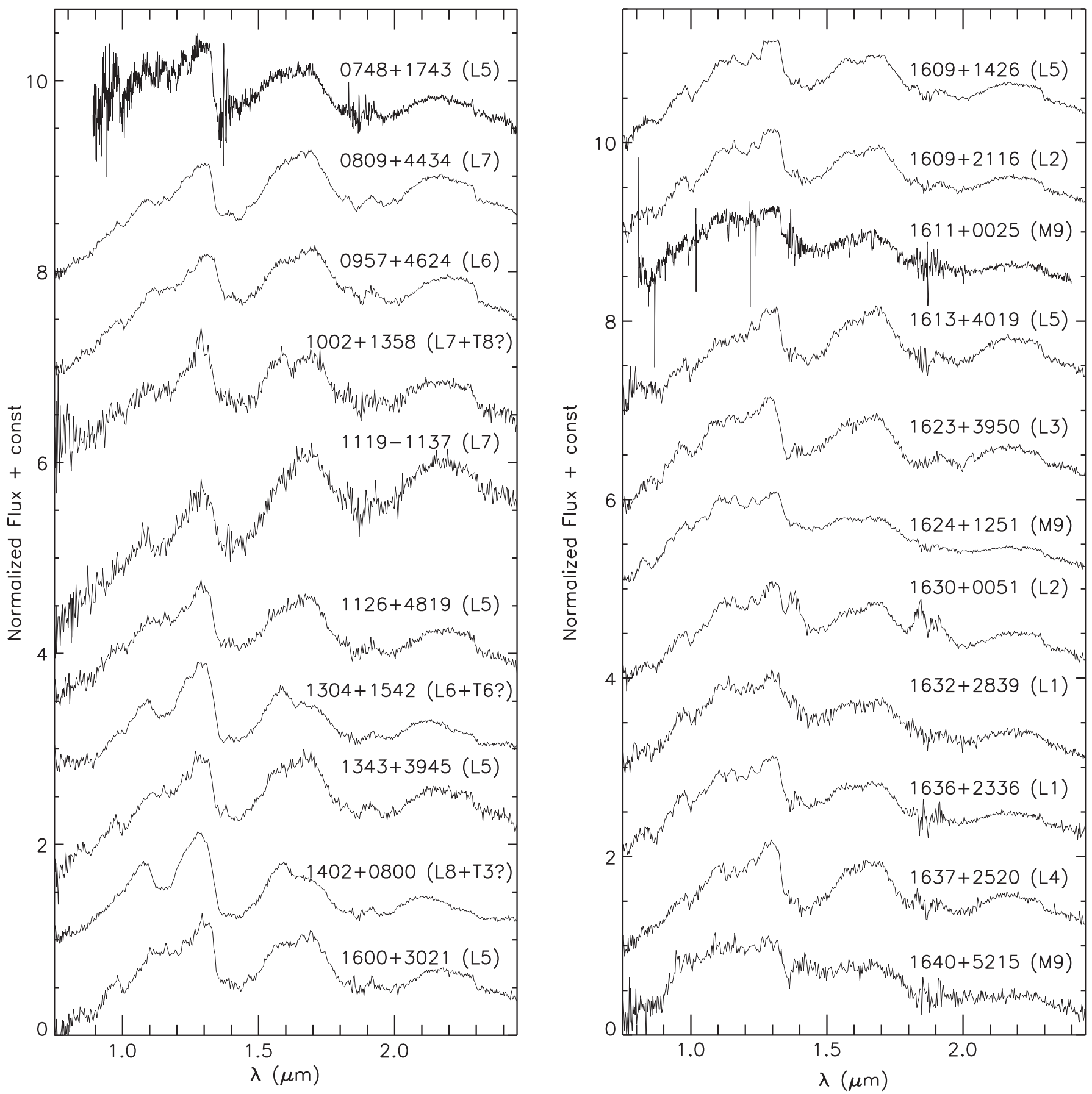

Figure 3. FIRE $(0748+1743,1611+0025 ; R \sim 400)$ and SpeX (all the rest; $R \sim 75-150)$ spectra of all of our reported ultra-cool dwarfs in order of right ascension. Spectral types are given in parentheses.

we have used the synthetic colors integrated from the spectra. We find that in a few cases the synthetic $z-J$ colors are bluer than 2.5 mag. As discussed in Section 3.3, this is likely the result of a flux overestimation bias for these faint targets, mostly in the 2MASS $J$ band. We discuss the normal, peculiar, and candidate binary ultra-cool dwarfs in our sample below.

A handful of objects have synthetic colors that are bluer than the $z-J=2.5 \mathrm{mag}$ color selection criterion. The SDSS and 2MASS photometry suggested that they were redder than $z-J=2.5 \mathrm{mag}$. However, their photometric $\mathrm{S} / \mathrm{Ns}$ from 2MASS and/or SDSS were low (see Section 3.3), and the synthetic photometry indicates that they are in fact bluer.

\subsection{Normal Ultra-cool Dwarfs}

We classify 17 of our candidates as normal L dwarfs, i.e., they do not have any readily apparent peculiarities based on their comparison to SpeX spectral standards. These objects are presented as black upward triangles in Figure 1(a). We find that a further 13 candidates are M7-M9 dwarfs. These were included in our program likely because the $i-z$ and $z-J$ colors of late-M dwarfs are close to the limits of our color selection criteria (Section 2.1), and because they may have been subject to flux overestimation bias at the $J$ band (Section 3.3). 

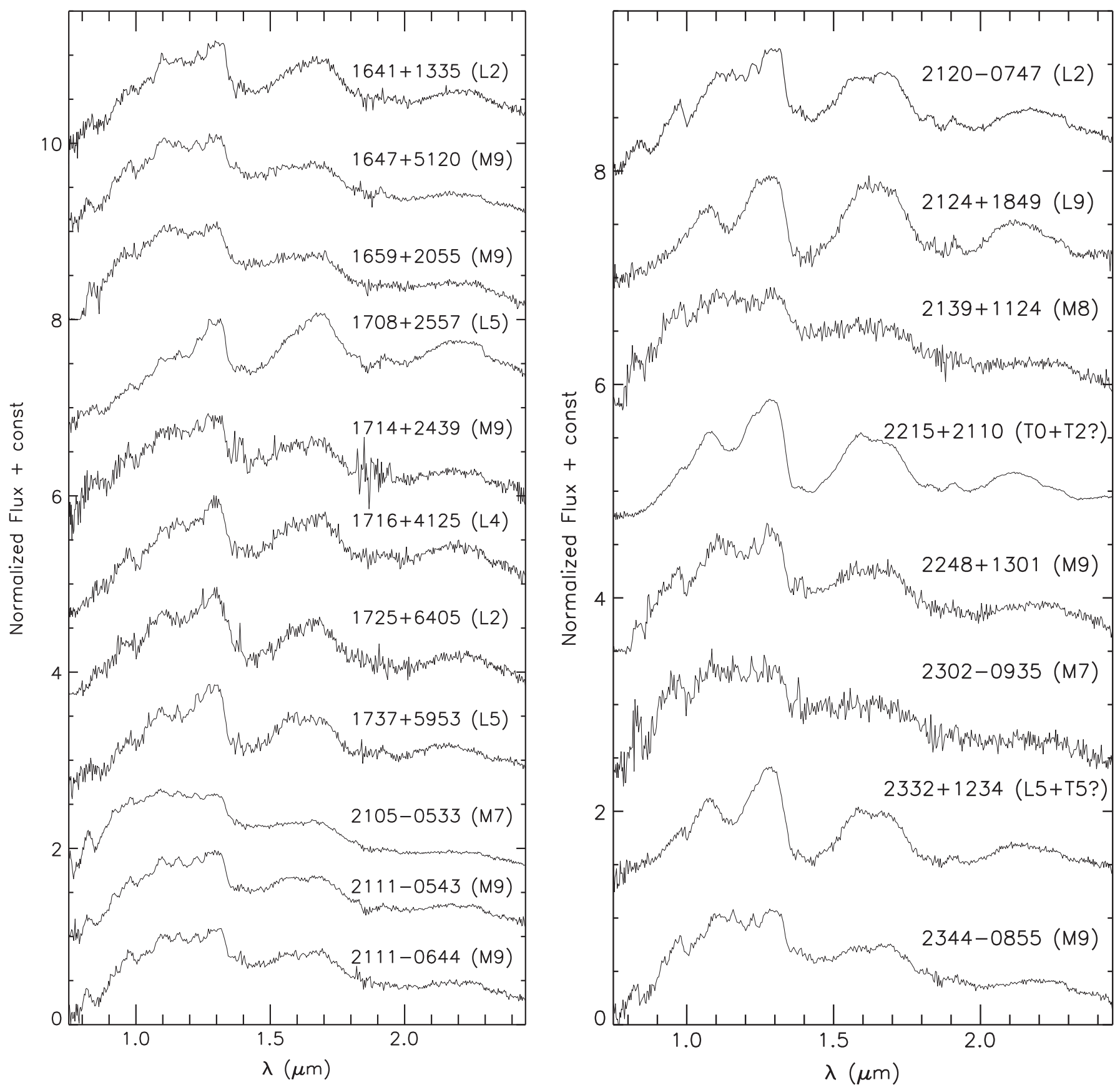

Figure 3. (Continued.)

\subsection{Peculiar L Dwarfs}

Various absorption features in the near-IR are gravitysensitive, hence, the low gravity of young brown dwarfs will result in line strengths that differ from those in older objects (e.g., Lucas et al. 2001; Gorlova et al. 2003; McGovern et al. 2004; Allers et al. 2007; Lodieu et al. 2008; Rice et al. 2010; Allers \& Liu 2013). Some of these features include the Na I $(1.138$ and $1.141 \mu \mathrm{m})$ and $\mathrm{K}_{\mathrm{I}}(1.169$ and $1.178 \mu \mathrm{m}$, 1.244 and $1.253 \mu \mathrm{m}$ ) doublets, $\mathrm{FeH}$ (bandheads at $0.990 \mu \mathrm{m}$ and $1.194 \mu \mathrm{m})$, and VO (1.05-1.08 and 1.17-1.22 $\mu \mathrm{m})$. Alkali lines are weaker at low gravity because of decreased pressure broadening. In low-resolution spectra these lines are often blended with other molecular features so we cannot obtain accurate measurements of their strengths. Metal hydride molecular features are also weaker at low gravity because of decreased opacity from these refractory species, while VO bands are stronger (see, e.g., Kirkpatrick et al. 2006). The $1.17 \mu \mathrm{m} \mathrm{VO}$ band is not used as a gravity indicator at low resolution because it is blended with $\mathrm{K}$,, $\mathrm{FeH}$, and $\mathrm{H}_{2} \mathrm{O}$ (Allers \& Liu 2013). Collision-induced absorption from molecular hydrogen $\left(\mathrm{H}_{2} \mathrm{CIA}\right)$ also changes as a function of gravity, with lower collision rates in low-gravity objects imparting a triangular shape to the $H$ band.

Several prior analyses have introduced broadband measures to discern low-gravity from field-gravity objects. Allers \& Liu (2013) design several near-IR indices to measure the changing 
strengths of $\mathrm{FeH}, \mathrm{VO}$, and $\mathrm{K}_{\mathrm{I}}$ absorption and the slope of the $\mathrm{H}$-band continuum as a function of gravity by comparing 1-100 Myr M5-L7 members of young moving associations with field dwarfs. Canty et al. (2013) analyzed M9-L0 dwarfs to design an $\mathrm{H}_{2}(\mathrm{~K})$ index that measures the contribution of $\mathrm{H}_{2}$ CIA on the slope of the $K$-band continuum; Schneider et al. (2014) expand this index to the L dwarfs. Indices have the potential to offer a quantitative gravity classification, analogous to spectral classification. However, index measures depend on the spectral resolution of the data used to calibrate them, and our spectra are sufficiently distinct from those used in prior studies. In addition, most of the indices do not extend into the late-L dwarfs, and so are inadequate to classify some of our most interesting objects. Therefore, we do not adopt spectral indices as a default gravity classification scheme. However, we do check for consistency with applicable spectral indices whenever we note peculiarities in the spectra of our L and T candidates.

We note that some of the spectral features, in particular the strength of the $\mathrm{FeH}$ bands, the peakiness of the $H$-band continuum, and the redness of the near-IR SED, may also be attributable to high atmospheric dust content or thicker clouds, as discussed in Looper et al. (2008b) and Allers \& Liu (2013). High dust content itself may be linked to low gravity, so a clear distinction may not always be possible, especially at low spectral resolution.

Our assessment of peculiarity is based on two factors: (1) the deviation from the median $J-K_{s}$ colors for objects of the same spectral type, with $>2 \sigma$ outliers considered peculiar, or (2) high spectral similarity to objects that have previously been classified as peculiar. In two cases below (Sections 4.2.24.2.3), we find similarities to the spectra of objects previously classified as peculiar because they are young and/or dusty. In the remaining two cases (Sections 4.2.1 and 4.2.4) the assessment of peculiarity is based on the comparison to spectra of previously classified peculiar objects as well as the $J-K_{s}$ colors.

\subsubsection{MASS J11193254-1137466 (L7)}

The most interesting object uncovered by our crosscorrelation is $2 \mathrm{M} 1119-1137$. This object is one of the reddest objects published to date, with a synthetic $J-K_{s}=$ $2.62 \pm 0.15$ mag. Only the L7 dwarfs PSO J318.5338 -22.8603 (Liu et al. 2013) and ULAS J222711-004547 (Marocco et al. 2014) among free-floating brown dwarfs are known to be redder. From its low-resolution spectrum (Figure 4), we classify this object as an L7. The low signalto-noise prevents us from unambiguously determining if this object has low gravity. The peak of the $H$-band continuumthought to be sharpened at low surface gravity (e.g., Lucas et al. 2001; Allers \& Liu 2013) - is not very sharp. We measured the $H$-cont index of Allers \& Liu (2013) and found a value of 0.907 , which is $1.5 \sigma$ above the medan for L7 dwarfs, and similar to the $H$-cont indices of low-gravity objects. The authors note that very red L dwarfs with no youth signatures can still exhibit triangular $H$-band shapes and similarly high $\mathrm{H}$ cont indices. In summary, the $H$-cont index of 2M1119-1137 is consistent with it being a low-gravity object, but we cannot conclude from the index alone that it is definitely young.

In Figure 5 we compare 2M1119-1137 to the known very low gravity dwarfs 2MASSW J224431.67+204343.3 (L7.5; Looper et al. 2008a), WISE J174102.78-464225.5 (L7;
Schneider et al. 2014), and WISEP J004701.06+680352.1 (L7.5; Gizis et al. 2012). We see that 2M1119-1137 most closely matches W0047+6803 and also matches the redness of W1741-4642 but has a less peaked $H$ band and a shallower slope in the $K$ band. Although it is slightly redder, the shape of the $H$ and $K$ band of 2M1119-1137 also matches that of 2M $2244+2043$. The agreement with the spectra of other young L7-L7.5 dwarfs also indicates that 2M1119-1137 may be young. A decisive classification will require higher-S/N and/ or higher-resolution spectra than we presently have.

Further evidence that 2M1119-1137 may be young comes from its proper motion and photometric distance. By comparing the 2MASS and AllWISE positions, we estimate an annual proper motion of $-155 \pm 20$ mas in R.A. and $-101 \pm 17$ mas in decl. Given a $K_{s}$ absolute magnitude of $12.6 \pm 0.4 \mathrm{mag}$ for young L7 dwarfs or $12.5 \pm 0.4$ mag for field-age L7 dwarfs (calculated from the empirically determined $L_{\mathrm{bol}}-\mathrm{SpT}$ relationship and $K_{s}$ bolometric corrections from Filippazzo et al. 2015), the photometric parallax of $2 \mathrm{M} 1119-1137$ is $40 \pm 12$ or $38 \pm 12$ mas. The BANYAN II space motion estimation algorithm (Malo et al. 2013; Gagné et al. 2014) gives 2M1119-1137 a probability between $39 \%$ and $69 \%$ of being a TW Hydrae moving group member, depending on whether an arbitrary age or a $<1$ Gyr age is chosen as an input prior with the respective photometric parallax estimates. Confirmation of the association with the TW Hydrae group will require radial velocity and trigonometric parallax measurements.

Should 2M1119-1137 be confirmed as a member of the 7-13 Myr (Bell et al. 2015) TW Hydrae association (Webb et al. 1999), it will be its coolest and lowest-mass (5-6 $M_{\text {Jup }}$, based on evolutionary models by Allard et al. 2012) freefloating member. Only the planetary-mass companion $2 \mathrm{M}$ 1207b (Chauvin et al. 2004, 2005) is likely to be cooler.

\subsubsection{MASS J17081563+2557474 (L5)}

This object is determined to be a young L5 brown dwarf based on the decreased absorption of $\mathrm{K}_{\mathrm{I}}$ and $\mathrm{FeH}$ and the increased absorption of $\mathrm{H}_{2} \mathrm{O}$ in the $J$ band. Calculations of the spectral indices from Allers \& Liu (2013) and Schneider et al. (2014) also suggest that this object is a low-gravity brown dwarf. As seen in Figure 4, the strengths of the gravitysensitive features in the $J$ band and the shape of the $H$ band are more similar to the young L5 2MASS J23174712-4838501 (Kirkpatrick et al. 2010), although the observed spectrum is still slightly redder than the comparison spectrum.

\subsubsection{MASS J16135698+4019158 (L5)}

While this object is peculiarly red, it does not exhibit the features of a low-gravity object. As seen in Figure 4, the object has normal absorption strengths, aside from $\mathrm{H}_{2} \mathrm{O}$, and is more similar to the red L5 dwarf 2M 2351+3010 published in Kirkpatrick et al. (2010). There is also strong FeH absorption. The authors speculate that $2 \mathrm{M} 2351+3010$ is actually an older object that simply has a higher dust content. Since our object seems very similar in nature, we adopt this explanation as well.

\subsubsection{MASS J17251557+6405005 (L2)}

$2 \mathrm{M} 1725+6405$ is a peculiarly blue L2 dwarf (Figure 4). This object was found in our cross-correlation but it was not part of our high-priority sample. Peculiarly blue L dwarfs have often been classified as metal-poor (e.g., Burgasser et al. 2003; 

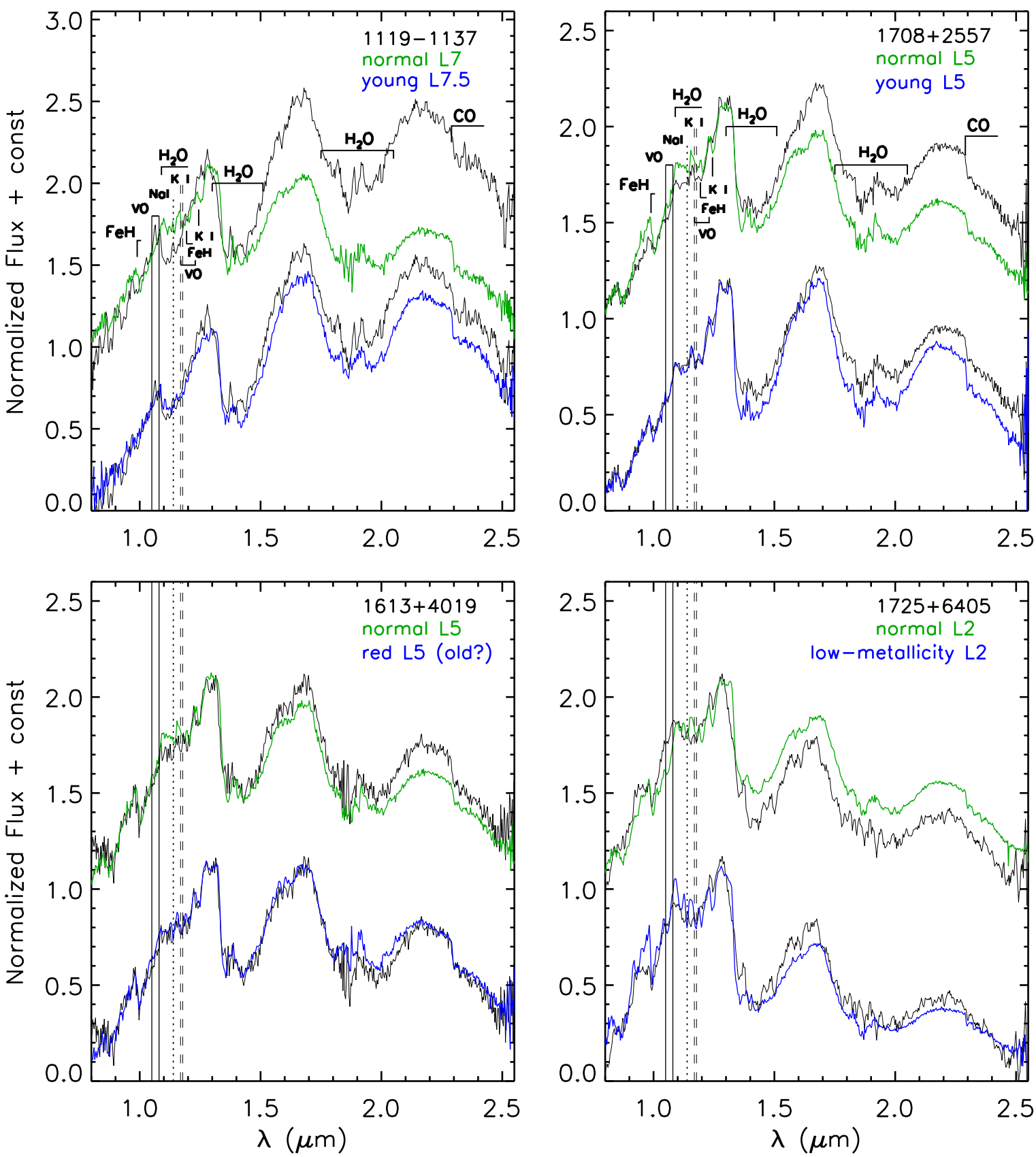

Figure 4. Spectra of the four peculiar objects identified in this work. In each case, the spectrum of the candidate is compared to the spectrum of a normal object of the same spectral type, and to the spectrum of a peculiar object of the nearest spectral type. The comparison spectra from left to right and top to bottom are L7 (2MASS J0028208+224905; Burgasser et al. 2010) and L7.5 young (2MASS J22443167+2043433; Looper et al. 2008a); L5 (2MASS J01550354+0950003; Burgasser et al. 2010) and L5 pec (2MASS J23174712-4838501; Kirkpatrick et al. 2010); L5 (2MASS J01550354+0950003; Burgasser et al. 2010) and L5 pec (2MASS J23512200+3010540; Kirkpatrick et al. 2010); L2 (2MASS J13054019-2541059; Burgasser et al. 2007) and L2 pec (2MASS J14313097+1436539; Sheppard \& Cushing 2009).

Burgasser 2004), with their blue near-IR colors dictated by increasingly strong collision-induced hydrogen absorption over 1.5-2.5 $\mu \mathrm{m}$. Metal-poor L dwarfs, or L subdwarfs, also show strong metal-hydride absorption. However, the FeH Wing-Ford band at $0.99 \mu \mathrm{m}$ in $2 \mathrm{M} 1725+6405$ is weak compared to the standard, which suggests that the $2 \mathrm{M} 1725+6405$ is blue likely because it is unusually dust-poor.

It is also possible that $2 \mathrm{M} 1725+6405$ may be an unresolved $\mathrm{L}+\mathrm{T}$ dwarf binary, with the $J$ band flux enhanced by the T dwarf component. We consider unresolved binarity in the next Section (4.3). Unlike all of the candidate binaries discussed in
Section 4.3, we actually do not find a better binary template fit for $2 \mathrm{M} 1725+6405$. We therefore conclude that this L2 dwarf is intrinsically blue.

\subsection{Brown Dwarfs with Composite Spectral Types}

Several of the objects show peculiarities that do not readily match those found in other individual objects. Instead, they more closely resemble combination spectra of $\mathrm{L}$ and $\mathrm{T}$ dwarfs. Burgasser (2007) and Burgasser et al. (2010) developed a technique that enables one to infer the spectral types of the individual components of a candidate unresolved binary by a 


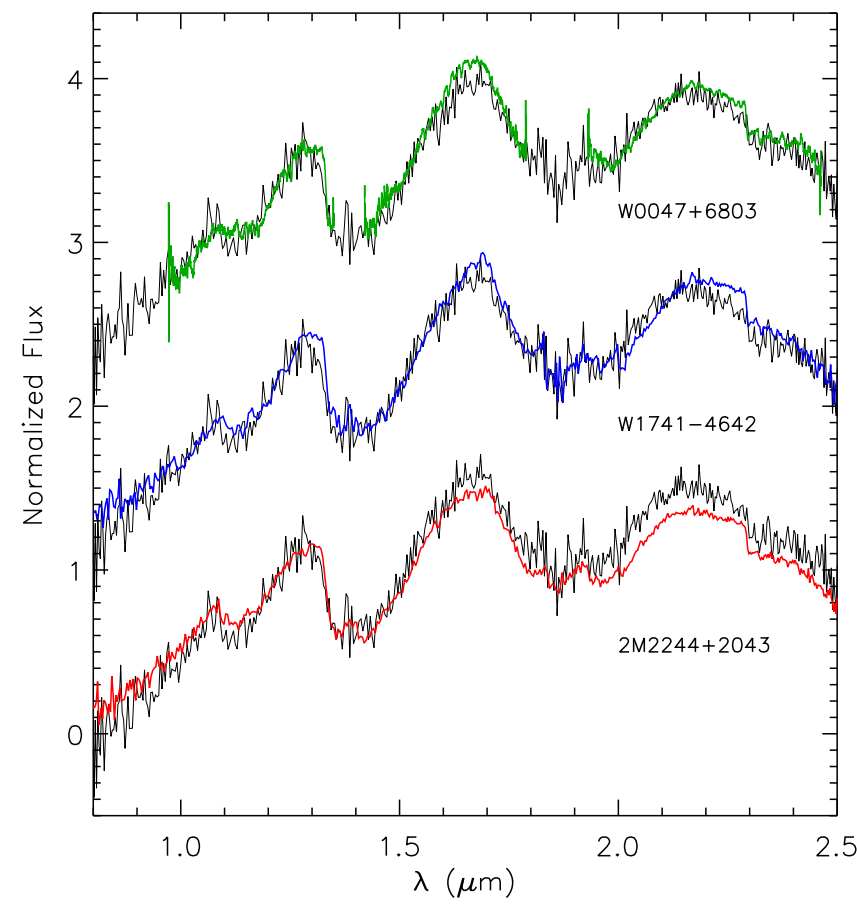

Figure 5. Comparison of the SpeX prism spectrum of 2M1119-1137 (black) with low-resolution spectra of other young L7-L7.5 dwarfs: WISEP J004701.06+680352.1 (L7.5 (pec); Gizis et al. 2012), WISE J174102.78 -464225.5 (L7 (pec); Schneider et al. 2014), and 2MASSW J224431.67 +204343.3 (L7.5 (pec); Kirkpatrick et al. 2010).

goodness-of-fit comparison to a library of spectral template combinations. We adopt this technique in a simple form, by creating combination templates from the set of single $\mathrm{L}$ and $\mathrm{T}$ dwarf standards from the SpeX Prism Library. Unlike Burgasser et al. (2010), we do not create a large list of templates built on the entire population of $\mathrm{L}$ and $\mathrm{T}$ dwarfs with available SpeX spectra. Nonetheless, we find that our simple approach gives sufficient indication of whether a brown dwarf displays a composite spectral signature and produces approximate spectral types for the components.

Our composite template spectra are constructed by normalizing all of the standard single brown dwarfs over the same wavelength range $(1.2-1.3 \mu \mathrm{m}$; chosen because it is relatively free of absorption features), scaling them to their absolute spectral-type dependent magnitudes given by the polynomials in Table 14 of Dupuy \& Liu (2012), and summing the pairs of resulting spectra. We compute the $\chi^{2}$ over most of the $0.8-2.5 \mu \mathrm{m}$ region, excluding ranges of strong water absorption $(1.35-1.45$ and $1.8-2.0 \mu \mathrm{m})$. In all cases, the $\chi^{2}$ is greater than one but this is to be expected as we are only testing the fit to templates created from one object of each spectral type. We have classified an object as a likely spectral type composite-a potential binary-if the $\chi^{2}$ of the dual-template spectral fit is significantly lower than the $\chi^{2}$ of the single-template fit. Each of the $\chi^{2}$ values has been calculated over the entire $0.8-2.5 \mu \mathrm{m}$ region, minus the water absorption bands.

In addition to template fitting, we have analyzed the spectral indices defined specifically for SpeX prism spectra in Burgasser et al. (2010) for all our binary candidates, and we report the strength of their candidate binarity. We have also analyzed the SpeX prism spectral indices from Bardalez Gagliuffi et al. (2014), but because the binary index selection criteria in that work were not designed for late-L to early- $\mathrm{T}$ dwarfs, we only report the results where applicable.

We note that while brown dwarfs displaying combination spectral signatures have until recently been considered to all be unresolved binaries, they can also be highly variable brown dwarfs with photospheres that display two distinct temperature components. Recent examples include the T1.5 dwarf 2MASS $\mathrm{J} 21392676+0220226$, suggested to be a strong L8.5 + T3.5 spectral binary candidate by Burgasser et al. (2010) but identified as a $J$-band variable that is unresolved in HST images (Radigan et al. 2012), or the T dwarfs 2MASS J13243559 +6358284 (T2.5) and SDSS J151114.66+060742.9 (T2), identified as binary candidates (Burgasser et al. 2010; Geißler et al. 2011) but that are also unresolved in HST and are variable (Metchev et al. 2015). Therefore, while the objects discussed in this section are considered candidate unresolved binaries, they are also strong candidates for photometric variables.

\subsubsection{MASS J13043568+1542521 (L6+T6?)}

This object is one of several that is best fit by a binary combination template. As seen in Figure 6, the best fit single brown dwarf (T0) does not match the features of 2M 1304 +1542 . The $Y-/ J$-band ratio is lower than any of the closest standard objects and the $H$ band has a dip at $\sim 1.65 \mu \mathrm{m}$. The $K$ band does not have differences that are as pronounced as in the other bands, though it is slightly redder than the standard object. In fitting this object with a binary template, we find that the best fit is a combination of an L6 and a T6 brown dwarf. The $Y$-/J-band ratio and the $K$-band flux more closely resemble the object spectrum. The contribution of the methane break in the cooler brown dwarf at $1.6 \mu \mathrm{m}$ also reproduces the dip in the $H$ band well. Further evidence that this object is a binary comes from the analysis of spectral indices identified in Burgasser et al. (2010) and Bardalez Gagliuffi et al. (2014). 2M 1304 +1542 satisfies 4 of the 6 binary index selection criteria given in Table 5 of Burgasser et al. (2010) and 10 of the 12 selection criteria in Table 4 of Bardalez Gagliuffi et al. (2014), making this a strong binary candidate.

\subsubsection{MASS J14025564+0800553 (L8+T5?)}

The spectrum of $2 \mathrm{M} 1402+0800$ also shows distinctive composite characteristics. While the $Y$-/J-band ratio is not significantly dissimilar from the closest single brown dwarf spectrum, the $H$ and $K$ bands are more similar to an L8+T5 binary. Figure 6 shows that the shape and relative flux of all three 2MASS bandpasses are very well reproduced by the $\mathrm{L} / \mathrm{T}$ binary template. Most importantly, the dip in the $H$ band is well reproduced by the contribution of the methane in the T dwarf. This object passes all six of the binary index selection criteria of Burgasser et al. (2010), which makes it a strong binary candidate.

\subsubsection{MASS J17373467+5953434 (L4+T5?)}

This object is classified as having an L4+T5 composite spectrum. As seen in Figure 6, an L5 spectrum matches 2M $1737+5953$ well in the $Y$ and $J$ bands but is a very poor match to the $H$ and $K$ bands. The observed spectrum shows signs of methane absorption at 1.6 and $2.2 \mu \mathrm{m}$ which is indicative of having a $\mathrm{T}$ dwarf secondary component. The binary index selection criteria from Burgasser et al. (2010) were not designed for mid-L dwarfs so we analyzed the spectral indices 


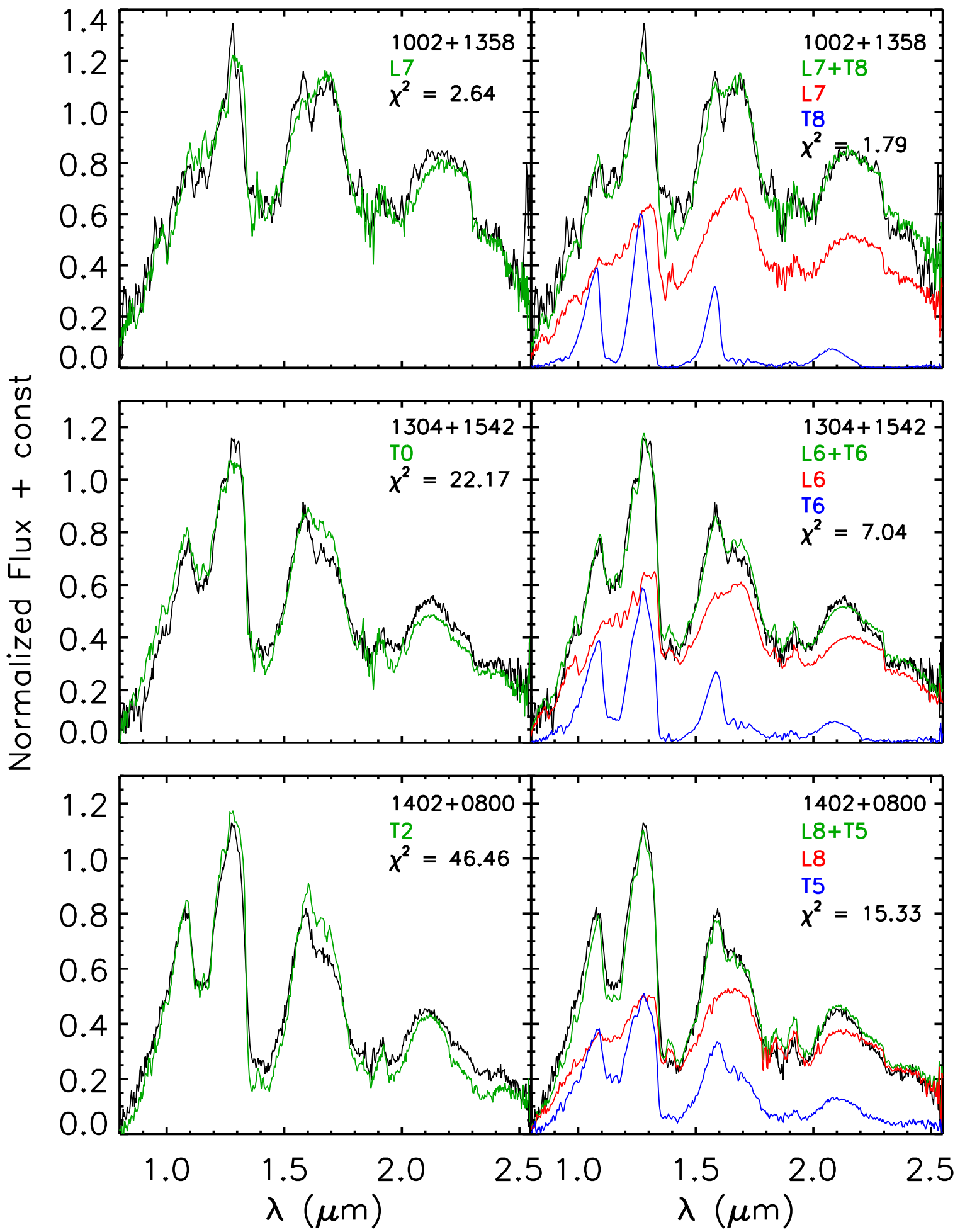

Figure 6. Spectra of all objects identified as candidate unresolved binaries (or photometric variables). The left panels show comparisons to the spectra (in green) that fit the $0.95-1.35 \mu \mathrm{m}$ continuum best: i.e., as done for spectral typing of the individual objects in Sections 4.1-4.2. The right panels show the two-component templates (also in green) that fit best over $0.8-2.5 \mu \mathrm{m}$; the individual component contributions are shown in red and blue. The quoted $\chi^{2}$ values are the smallest ones for, respectively, single and binary template fits over the entire $0.8-2.5 \mu \mathrm{m}$ range, as was done in Section 4.3. The comparison spectra from left to right and top to bottom are: L7 (2MASS J0028208+224905; Burgasser et al. 2010) and T8 (2MASS J04151954-0935066; Burgasser et al. 2004); T0 (2MASS J12074717+0244249; Looper et al. 2007), L6 (2MASS J10101480-0406499; Reid et al. 2006), and T6 (2MASS J16241436+0029158; Burgasser et al. 2006a); T2 (2MASS J12545393-0122474; Burgasser et al. 2004), L8 (2MASS J16322911+1904407; Burgasser 2007), and T5 (2MASS J15031961+2525196; Burgasser et al. 2004). The comparison spectra from left to right and top to bottom are: L5 (2MASS J08350622+1953050; Chiu et al. 2006), L4 (2MASS J21580457-1550098; Kirkpatrick et al. 2010), and T5 (2MASS J15031961+2525196; Burgasser et al. 2004); T1 (2MASS J01514155+1244300; Burgasser et al. 2004), T0 (2MASS J12074717+0244249; Looper et al. 2007), and T2 (2MASS J12545393-0122474; Burgasser et al. 2004); L5 (2MASS J08350622+1953050; Chiu et al. 2006) and T5 (2MASS J15031961 +2525196 ; Burgasser et al. 2004). 


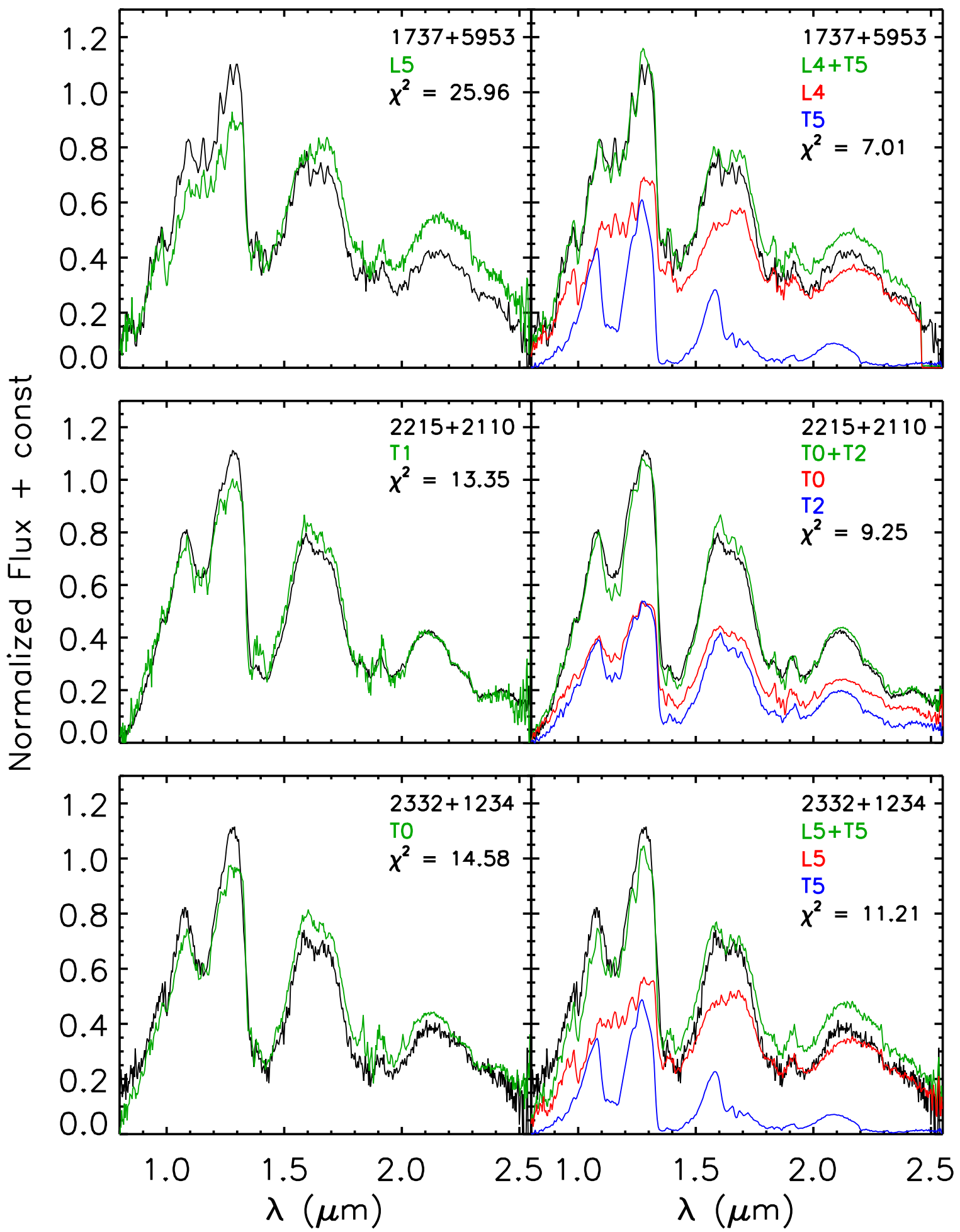

Figure 6. (Continued.)

from Bardalez Gagliuffi et al. (2014) instead. Because this object only passes 4 of the 12 selection criteria from Bardalez Gagliuffi et al. (2014), it is only a weak binary candidate.

\subsubsection{MASS J23322678+1234530 (L5+T5?)}

While $2 \mathrm{M} 2332+1234$ is best fit in the $J$ band by a scaled T0 spectrum, the $H$ and $K$ bands clearly do not appear to belong to a T0 dwarf. The $H$ band shows evidence of methane absorption at $1.6-1.8 \mu \mathrm{m}$ but there is less of a presence of $\mathrm{CH}_{4}$ in the $K$ band. This points to a composite L/ T spectrum similar to SDSS J151114.66+060742.9 presented in Burgasser et al. (2010). The methane absorption features are best fit by an L $5+\mathrm{T} 5$ template; however, the continuum of our observed spectrum is still slightly bluer at longer wavelengths. This object passes four of the binary index 
selection criteria of Burgasser et al. (2010), which makes it a strong binary candidate.

\subsubsection{MASS J10020752+1358556 (L7+T8?)}

This object is tentatively classified as having a composite spectrum. As seen in Figure 6, 2M $1002+1358$ has a large dip in flux in the $H$ band at the location of the $\mathrm{CH}_{4}$ absorption feature that is usually present in a $\mathrm{T}$ dwarf, and has much more water and methane absorption in the $J$ band than a typical $\mathrm{L}$ dwarf. The $K$ band, however, seems to be similar to an L4-L6 dwarf. This suggests a composite spectral type. There is a much greater difference between $\mathrm{L}$ and T dwarfs in the $J$ - and $H$-band features than there is in the $K$-band features, and therefore, the $K$ band of a combined binary spectrum can look like it belongs to an $\mathrm{L}$ dwarf, whereas the $J$ and $H$ bands will appear to have a contribution from both binary components. The large dip in $\mathrm{H}$ band flux may also be the result of an extraneous signal in the raw spectrum of the object, as it has an atypical shape compared to that of a feature usually associated with $\mathrm{CH}_{4}$. However, the spectrum of the telluric calibration star does not exhibit the same behavior, while the feature is apparent in most of the individual spectra of this object, even if at low $\mathrm{S} / \mathrm{N}$. This suggests that the feature may be real, even if we cannot fully exclude a random variation due to noise. Analyzing the spectral indices does not shed any light on the true nature of this object, as it only passes 4 of the 12 binary index selection criteria from Bardalez Gagliuffi et al. (2014), making it a weak binary candidate.

\subsection{6. $2 M A S S ~ J 22153705+2110554(T 0+T 2$ ?)}

The T dwarf $2 \mathrm{M} 2215+2110$ is a new discovery in the SDSS footprint. Some of the features in the spectrum of $2 \mathrm{M} 2215$ +2110 are ambiguous as to their origin. While the $J$ and $K$ bands more closely resemble an early-T dwarf, the $H$ band has a clear dearth of flux. The overall shape of this band might be explained by the presence of a slightly later-type $T$ dwarf secondary component than the primary, but the lack of flux still persists in the binary template spectrum. Several features, such as the $\mathrm{FeH}$ feature at $0.99 \mu \mathrm{m}$, do match a $\mathrm{T} 0+\mathrm{T} 2$ composite spectrum. However, the $\mathrm{H}_{2} \mathrm{O}+\mathrm{CH}_{4}$ absorption between 1.1 and $1.2 \mu \mathrm{m}$ is much stronger in the binary composite template than in the observed spectrum. The spectral indices also do not help us with this object-only two of the index selection criteria from Burgasser et al. (2010) are passed, which makes this object a weak binary candidate.

\section{DISCUSSION}

Our search was aimed at discovering peculiar $\mathrm{L}$ or $\mathrm{T}$ dwarfs, with priority in this first iteration placed on unusually red objects. Overall, we have observed and identified 10 peculiar or binary L dwarfs, 16 normal $\mathrm{L}$ dwarfs, $1 \mathrm{~T}$ dwarf, and $13 \mathrm{M}$ dwarfs. The latter had been misidentified as candidate $\mathrm{L}$ or $\mathrm{T}$ dwarfs because of low S/N photometry.

The total fraction of objects in an unbiased sample of brown dwarfs with $J-K_{s}$ colors $>2 \sigma$ from the mean color at a given spectral type - the criterion used for detecting photometrically peculiar L and T dwarfs in Faherty et al. (2009)-is expected to be $4.6 \%$. Faherty et al. (2009) report a somewhat larger fraction, 5.8\%, of peculiar objects among the 1268 M7-T8 dwarfs in their sample. The small discrepancy arises from an apparent non-gaussianity of the $J-K_{s}$ color distribution: they have nearly twice as many red outliers than blue outliers.

Only three of our L dwarfs are peculiarly red or dusty, and an equal number of our discoveries are in fact peculiarly blue. While at face value this does not indicate a higher success rate in finding peculiarly red objects than in a random sample of field brown dwarfs, we have at present followed up only a small number (40) of our total candidate sample (314). The 40 objects presented here comprise roughly equal numbers of high- (22) and low-priority (18) objects: a circumstance of weather and observational constraints. It is possible that the larger high-priority sample (178 candidates) will reveal a higher incidence rate of unusually red objects.

We do find, however, that our present prioritization strategy reveals a larger fraction of unusual objects-including not only peculiar L dwarfs but also candidate unresolved binaries that are not color outliers in $J-K_{s}$ but are unusually red in $z-J$ - among the high-priority candidates. Eight of the 22 objects in the high-priority sample are peculiar or candidate binaries versus 2 of the 18 in the low-priority sample. The difference between the two is statistically significant at the $96 \%$ level. It indicates that combinations of optical and infrared colors, such as employed here, can successfully discern even moderate peculiarities in ultra-cool dwarfs. Table 3 summarizes the peculiarities of each object from spectral comparison and synthetic colors.

Because $\mathrm{L}$ and $\mathrm{T}$ dwarfs are brighter in the $3-5 \mu \mathrm{m}$ wavelength range, we investigated whether the $J-K_{s}$ color outliers also have unusual colors at these wavelengths. We find that $\mathrm{L}$ dwarfs with the very reddest $J-K_{s}$ colors are clearly distinguishable from the locus of L dwarfs on a $J-K_{s}$ versus $H-W 2$ and $J-K_{s}$ versus $W 1-W 2$ diagram (Figure 7) mainly because of their red near-IR colors. They stand out in their $J-K_{s}$ and $H-W 2$ colors but not significantly in their $W 1-W 2$ colors. T dwarfs with peculiarly red $J-K_{s}$ colors are only marginally redder in $H-W 2$ and $W 1-W 2$, and the peculiarly blue $\mathrm{L}$ or $\mathrm{T}$ dwarfs are not distinguishable from the normal population with the exception of the blue L dwarf discovered in this work (2MASS J17251557+6405005).

\section{CONCLUSIONS}

We performed a color-selected search for peculiar $\mathrm{L}$ and $\mathrm{T}$ dwarfs, focusing primarily on peculiarly red objects, and demonstrated that with the proper selection criteria, we can identify unusual $\mathrm{L}$ and $\mathrm{T}$ dwarf candidates in large photometric surveys in the absence of spectral type information. With follow-up spectroscopy, we can verify the unusual properties and begin to discern their underlying cause. This is particularly advantageous for finding isolated objects that are analogous to the typically very red directly imaged extrasolar planets in order to study their atmospheric characteristics at higher fidelity. We had a high success rate in discovering either peculiar L dwarfs or candidate unresolved binaries in our prioritized sample, and discovered one of the reddest $\mathrm{L}$ dwarfs known to date. This new red L7 dwarf is a potential TW Hydrae member, and if confirmed, would make it the coolest and least massive free-floating object in the association. We note that even after many searches for T dwarfs in the SDSS and 2MASS catalogs, we still uncovered a new T dwarf among the $\sim 13 \%$ fraction of candidates that we have spectroscopically characterized so far. These discoveries attest to the power of simultaneous positional and color cross-correlations across 


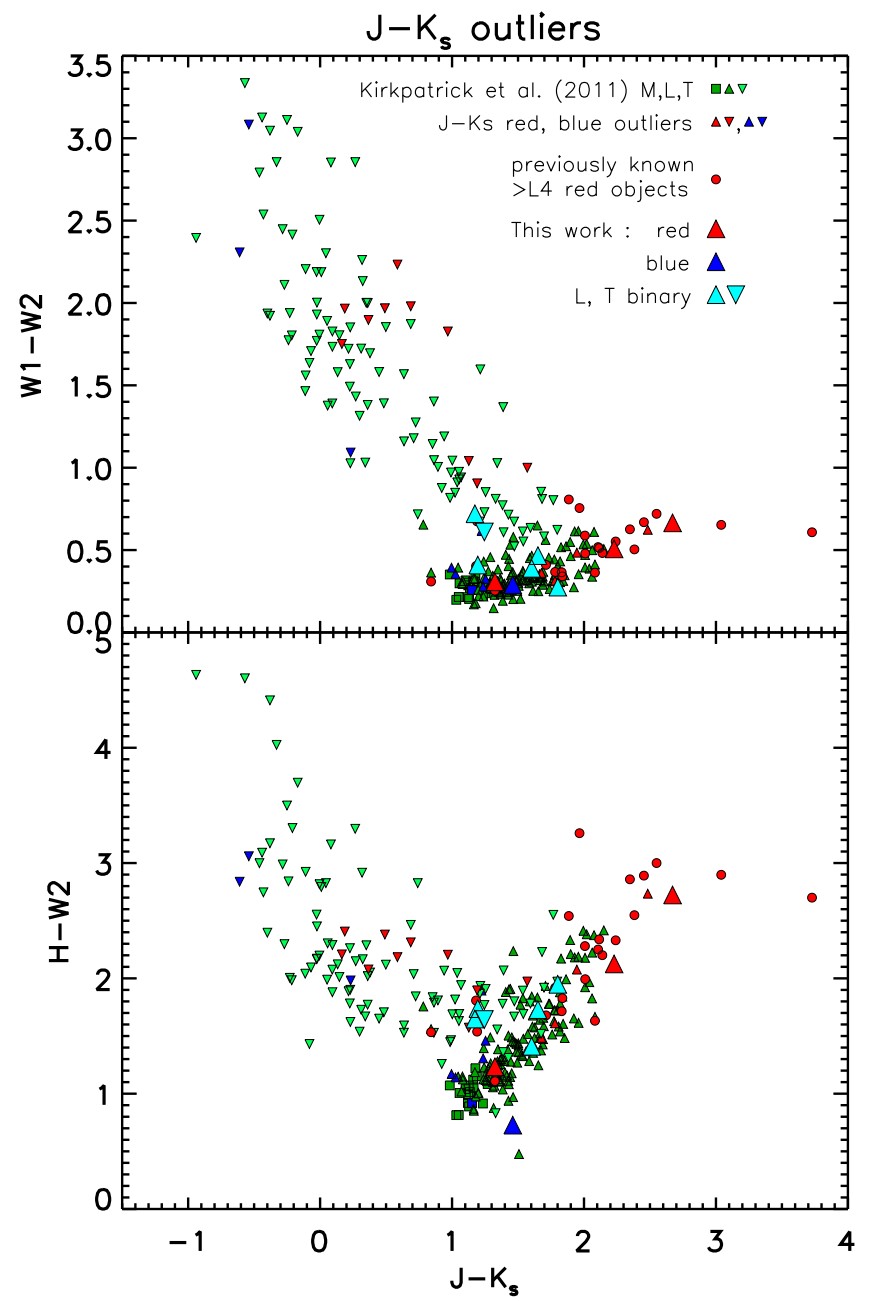

Figure 7. Photometric color-color diagrams of objects from Kirkpatrick et al. (2011). Upward and downward triangles denote L and T dwarfs, respectively. Red symbols denote objects with $J-K_{s}$ colors $>2 \sigma$ redder than the mean for their spectral type (Faherty et al. 2009, 2013). Blue symbols denote objects that are $>2 \sigma$ bluer. Large symbols represent peculiar objects identified in this work. Red circles indicate the previously known red brown dwarfs with spectral types of L4 and later.

photometric databases - as performed here, in Metchev et al. (2008), in Geißler et al. (2011), and now enabled with the Virtual Astronomical Observatory-over color-only searches on individual databases that are then positionally compared to other databases. At the same time, the discovery of only a single new T dwarf in our characterized sample indicates that the census of T dwarfs (132) in SDSS is nearly complete.

This work was supported by the NASA Astrophysical Data Analysis Program through award No. NNX11AB18G to S.M. at Stony Brook University and by an NSERC Discovery grant to S.M. at The University of Western Ontario. Part of the data were obtained with the Magellan-Baade $6.5 \mathrm{~m}$ telescope under program CN2012A-54. Support for R.K. is provided from Fondecyt Reg. No. 1130140 and by the Ministry of Economy, Development, and Tourisms Millennium Science Initiative through grant IC12009, awarded to The Millennium Institute of Astrophysics (MAS). The authors wish to recognize and acknowledge the very significant cultural role and reverence that the summit of Mauna Kea has always had within the indigenous Hawaiian community. We are most fortunate to have the opportunity to conduct observations from this mountain. This paper includes data gathered with the $6.5 \mathrm{~m}$ Magellan Telescopes located at Las Campanas Observatory, Chile. This publication makes use of data products from the Two Micron All Sky Survey, which is a joint project of the University of Massachusetts and the Infrared Processing and Analysis Center/California Institute of Technology, funded by the National Aeronautics and Space Administration and the National Science Foundation. Funding for SDSS-III has been provided by the Alfred P. Sloan Foundation, the Participating Institutions, the National Science Foundation, and the U.S. Department of Energy Office of Science. The SDSS-III web site is http://www.sdss3.org/. SDSS-III is managed by the Astrophysical Research Consortium for the Participating Institutions of the SDSS-III Collaboration including the University of Arizona, the Brazilian Participation Group, Brookhaven National Laboratory, Carnegie Mellon University, University of Florida, the French Participation Group, the German Participation Group, Harvard University, the Instituto de Astrofisica de Canarias, the Michigan State/Notre Dame/ JINA Participation Group, Johns Hopkins University, Lawrence Berkeley National Laboratory, Max Planck Institute for Astrophysics, Max Planck Institute for Extraterrestrial Physics, New Mexico State University, New York University, Ohio State University, Pennsylvania State University, University of Portsmouth, Princeton University, the Spanish Participation Group, University of Tokyo, University of Utah, Vanderbilt University, University of Virginia, University of Washington, and Yale University. This publication makes use of data products from the Wide-field Infrared Survey Explorer, which is a joint project of the University of California, Los Angeles, and the Jet Propulsion Laboratory/California Institute of Technology, funded by the National Aeronautics and Space Administration. This research has made use of data obtained from or software provided by the US Virtual Astronomical Observatory, which is sponsored by the National Science Foundation and the National Aeronautics and Space Administration. We thank our referee, Jackie Faherty, for her insightful critique.

Note added in proof. During the review process, Marocco et al. (2015) presented 2MASS $1304+1542$ as a result of their UKIDSS search for brown dwarfs. The authors also identified this object as a strong binary candidate with a composite L7.0 $+\mathrm{T} 5.5$ spectrum giving the lowest $\chi^{2}$ value. Our assessment is in good agreement with these results.

Facilities: IRTF (SpeX spectrograph), Magellan:Baade (FIRE spectrograph).

\section{REFERENCES}

Ahn, C. P., Alexandroff, R., Allende Prieto, C., et al. 2012, ApJS, 203, 21 Allard, F., Homeier, D., \& Freytag, B. 2012, RSPTA, 370, 2765 Aller, K. M., Kraus, A. L., Liu, M. C., et al. 2013, ApJ, 773, 63 Allers, K. N., Jaffe, D. T., Luhman, K. L., et al. 2007, ApJ, 657, 511 Allers, K. N., \& Liu, M. C. 2013, ApJ, 772, 79

Bardalez Gagliuffi, D. C., Burgasser, A. J., Gelino, C. R., et al. 2014, ApJ, 794, 143

Bell, C. P. M., Mamajek, E. E., \& Naylor, T. 2015, MNRAS, 454, 593

Bochanski, J. J., Hennawi, J. F., Simcoe, R. A., et al. 2009, PASP, 121, 1409 Bonnefoy, M., Boccaletti, A., Lagrange, A.-M., et al. 2013, A\&A, 555, A107 Burgasser, A. J. 2004, ApJL, 614, L73 Burgasser, A. J. 2007, ApJ, 659, 655

Burgasser, A. J., Burrows, A., \& Kirkpatrick, J. D. 2006a, ApJ, 639, 1095 Burgasser, A. J., Cruz, K. L., Cushing, M., et al. 2010, ApJ, 710, 1142 Burgasser, A. J., Geballe, T. R., Leggett, S. K., Kirkpatrick, J. D., \& Golimowski, D. A. 2006b, ApJ, 637, 1067 
Burgasser, A. J., Kirkpatrick, J. D., Burrows, A., et al. 2003, ApJ, 592, 1186 Burgasser, A. J., Liu, M. C., Ireland, M. J., Cruz, K. L., \& Dupuy, T. J. 2008, ApJ, 681, 579

Burgasser, A. J., Looper, D. L., Kirkpatrick, J. D., \& Liu, M. C. 2007, ApJ, 658,557

Burgasser, A. J., McElwain, M. W., Kirkpatrick, J. D., et al. 2004, AJ, 127,2856

Canty, J. I., Lucas, P. W., Roche, P. F., \& Pinfield, D. J. 2013, MNRAS, 435,2650

Chauvin, G., Lagrange, A.-M., Dumas, C., et al. 2004, A\&A, 425, L29

Chauvin, G., Lagrange, A.-M., Dumas, C., et al. 2005, A\&A, 438, L25

Chiu, K., Fan, X., Leggett, S. K., et al. 2006, AJ, 131, 2722

Cruz, K. L., Kirkpatrick, J. D., \& Burgasser, A. J. 2009, AJ, 137, 3345

Cushing, M. C., Vacca, W. D., \& Rayner, J. T. 2004, PASP, 116, 362

Cutri, R. 2006, 2MASS All-Sky Data Release Explanatory Supplement: Full Survey Image Atlas and Source "Reject" Tables; Cautionary Notes, http:// www.ipac.caltech.edu/2mass/releases/allsky/doc/seca2_1c.html, [Online; accessed 26 October 2013]

Dupuy, T. J., \& Liu, M. C. 2012, ApJS, 201, 19

Faherty, J. K., Burgasser, A. J., Cruz, K. L., et al. 2009, AJ, 137, 1

Faherty, J. K., Burgasser, A. J., Walter, F. M., et al. 2012, ApJ, 752, 56

Faherty, J. K., Rice, E. L., Cruz, K. L., Mamajek, E. E., \& Núñez, A. 2013, AJ, 145,2

Filippazzo, J. C., Rice, E. L., Faherty, J., et al. 2015, ApJ, 810, 158

Gagné, J., Lafrenière, D., Doyon, R., Malo, L., \& Artigau, É. 2014, ApJ, 783,121

Gagné, J., Lafrenière, D., Doyon, R., Malo, L., \& Artigau, É. 2015, ApJ, 798, 73

Geißler, K., Metchev, S., Kirkpatrick, J. D., Berriman, G. B., \& Looper, D. 2011, ApJ, 732, 56

Gizis, J. E., Faherty, J. K., Liu, M. C., et al. 2012, AJ, 144, 94

Golimowski, D. A., Leggett, S. K., Marley, M. S., et al. 2004, AJ, 127, 3516

Gorlova, N. I., Meyer, M. R., Rieke, G. H., \& Liebert, J. 2003, ApJ, 593, 1074

Horne, K. 1986, PASP, 98, 609

Kirkpatrick, J. D., Barman, T. S., Burgasser, A. J., et al. 2006, ApJ, 639, 1120 Kirkpatrick, J. D., Cushing, M. C., Gelino, C. R., et al. 2011, ApJS, 197, 19 Kirkpatrick, J. D., Looper, D. L., Burgasser, A. J., et al. 2010, ApJS, 190, 100 Kirkpatrick, J. D., Reid, I. N., Liebert, J., et al. 2000, AJ, 120, 447

Knapp, G. R., Leggett, S. K., Fan, X., et al. 2004, AJ, 127, 3553

Liu, M. C., Magnier, E. A., Deacon, N. R., et al. 2013, ApJL, 777, L20
Lodieu, N., Hambly, N. C., Jameson, R. F., \& Hodgkin, S. T. 2008, MNRAS, 383, 1385

Looper, D. L., Gelino, C. R., Burgasser, A. J., \& Kirkpatrick, J. D. 2008a, ApJ, 685,1183

Looper, D. L., Kirkpatrick, J. D., \& Burgasser, A. J. 2007, AJ, 134, 1162

Looper, D. L., Kirkpatrick, J. D., Cutri, R. M., et al. 2008b, ApJ, 686, 528

Lucas, P. W., Roche, P. F., Allard, F., \& Hauschildt, P. H. 2001, MNRAS, 326, 695

Luhman, K. L., \& Sheppard, S. S. 2014, ApJ, 787, 126

Lupton, R. H., Ivezic, Z., Gunn, J. E., et al. 2002, Proc. SPIE, 4836, 350

Malo, L., Doyon, R., Lafrenière, D., et al. 2013, ApJ, 762, 88

Marley, M. S., Saumon, D., Cushing, M., et al. 2012, ApJ, 754, 135

Marocco, F., Day-Jones, A. C., Lucas, P. W., et al. 2014, MNRAS, 439, 372

Marocco, F., Jones, H. R. A., Day-Jones, A. C., et al. 2015, MNRAS, 449, 3651

McGovern, M. R., Kirkpatrick, J. D., McLean, I. S., et al. 2004, ApJ, 600, 1020

McLean, I. S., Prato, L., McGovern, M. R., et al. 2007, ApJ, 658, 1217

Metchev, S. A., Heinze, A., Apai, D., et al. 2015, ApJ, 799, 154

Metchev, S. A., Kirkpatrick, J. D., Berriman, G. B., \& Looper, D. 2008, ApJ, 676, 1281

Press, W. H., Teukolsky, S. A., Vetterling, W. T., \& Flannery, B. P. 1992, Numerical Recipes in FORTRAN. The Art of Scientific Computing (Cambridge: Cambridge Univ. Press)

Radigan, J., Jayawardhana, R., Lafrenière, D., et al. 2012, ApJ, 750, 105

Rayner, J. T., Toomey, D. W., Onaka, P. M., et al. 2003, PASP, 115, 362

Reid, I. N., Lewitus, E., Burgasser, A. J., \& Cruz, K. L. 2006, ApJ, 639, 1114

Rice, E. L., Barman, T., Mclean, I. S., Prato, L., \& Kirkpatrick, J. D. 2010, ApJS, 186, 63

Robertson, J. G. 1986, PASP, 98, 1220

Schmidt, S. J., West, A. A., Hawley, S. L., \& Pineda, J. S. 2010, AJ, 139, 1808

Schneider, A. C., Cushing, M. C., Kirkpatrick, J. D., et al. 2014, AJ, 147, 34

Sheppard, S. S., \& Cushing, M. C. 2009, AJ, 137, 304

Simcoe, R. A., Burgasser, A. J., Bernstein, R. A., et al. 2008, Proc. SPIE, 7014, 0

Vacca, W. D., Cushing, M. C., \& Rayner, J. T. 2003, PASP, 115, 389

Vrba, F. J., Henden, A. A., Luginbuhl, C. B., et al. 2004, AJ, 127, 2948

Webb, R. A., Zuckerman, B., Platais, I., et al. 1999, ApJL, 512, L63

Yasuda, N., Fukugita, M., Narayanan, V. K., et al. 2001, AJ, 122, 1104

Zhang, Z. H., Pokorny, R. S., Jones, H. R. A., et al. 2009, A\&A, 497, 619 